\title{
Arbeitszufriedenheit im internationalen Vergleich
}

Hanglberger, Dominik

Publication date:

2011

Document Version

Verlags-PDF (auch: Version of Record)

Link to publication

Citation for pulished version (APA):

Hanglberger, D. (2011). Arbeitszufriedenheit im internationalen Vergleich. (FFB-Diskussionspapier; Nr. 86). Forschungsinstitut Freie Berufe.

\section{General rights}

Copyright and moral rights for the publications made accessible in the public portal are retained by the authors and/or other copyright owners and it is a condition of accessing publications that users recognise and abide by the legal requirements associated with these rights.

- Users may download and print one copy of any publication from the public portal for the purpose of private study or research.

- You may not further distribute the material or use it for any profit-making activity or commercial gain

- You may freely distribute the URL identifying the publication in the public portal ?

Take down policy

If you believe that this document breaches copyright please contact us providing details, and we will remove access to the work immediately and investigate your claim. 


\section{Forschungsinstitut}

Freie Berufe

\section{Arbeitszufriedenheit im internationalen \\ Vergleich}

\section{Dominik Hanglberger}

FFB-Diskussionspapier Nr. 86

Januar 2010

\section{$\bowtie$ \\ LEUPFANA \\ UNIVERSITÄT LÜNEBURG}

\section{Fakultät II - Wirtschafts-, Verhaltens- und Rechtswissenschaften}

Postanschrift:

Forschungsinstitut Freie Berufe

Postfach 2440

21314 Lüneburg ffb@uni-lueneburg.de

http:/ /ffb.uni-lueneburg.de

Tel: +494131677-2051

Fax:+49 4131 677-2059 


\title{
Arbeitszufriedenheit im internationalen Vergleich
}

\author{
Dominik Hanglberger ${ }^{1}$
}

FFB-Diskussionspapier Nr. 86

Januar 2011

ISSN 0942-2595

1 Dipl.-Volksw. Dominik Hanglberger, Leuphana Universität Lüneburg, Fakultät Wirtschaft, Forschungsinstitut Freie Berufe (FFB), Professur ,Statistik und Freie Berufe', Scharnhorststr. 1, 21335 Lüneburg, Tel.: +49 4131 / 677-2051, Fax: +49 4131 / 677-2059, E-Mail: hanglberger@uni-leuphana.de, www.leuphana.de/ffb. 


\title{
Arbeitszufriedenheit im internationalen Vergleich \\ Dominik Hanglberger \\ FFB-Diskussionspapier Nr. 86, Januar 2011, ISSN 0942-2595
}

\section{Zusammenfassung}

Ziel dieses Beitrags ist es, Niveaus und Bestimmungsfaktoren der Arbeitszufriedenheit von abhängig Beschäftigten in Europa zu vergleichen. Dafür werden Daten aus dem European Working Conditions Survey (EWCS) 2005 für 31 europäische Staaten ausgewertet. Neben detaillierten Informationen über Art und Ausgestaltung der Arbeit liegen dafür Personen- und Haushaltsinformationen sowie objektive und subjektive Einkommensinformationen vor. Ordered-Probit Regressionsmodelle für alle 31 Länder sowie getrennte Schätzungen für fünf Ländergruppen mit unterschiedlichen Wohlfahrtsniveaus und unterschiedlicher Ausgestaltung der Wohlfahrtsstaaten zeigen, dass die Erklärungsmuster der Arbeitszufriedenheit nicht einheitlich sind. Über alle Länder zeigt sich ein starkes Gewicht der subjektiven Bewertung des Einkommens, die nur in Skandinavien und Kontinentaleuropa nicht die stärkste Einflussgröße darstellt. Bezüglich der Arbeitszeiten zeigt sich in Ländern mit niedrigerem Wohlstandsniveau ein geringerer negativer Einfluss, wenn Arbeitszeiten mit privaten Verpflichtungen kollidieren. In Großbritannien und Irland spielt die Sicherheit des Arbeitsplatzes eine größere Rolle als in den übrigen betrachteten Ländern. Die Autonomie bei der Organisation der Arbeitsaufgaben findet sich nur in Staaten mit hohem Wohlstandsniveau (Großbritannien, Irland, Kontinentaleuropa und Skandinavien) unter den zehn stärksten Einflüssen.

JEL: J28, J81

Schlagwörter: Arbeitszufriedenheit, internationaler Vergleich, Arbeitsbedingungen, Ordered-Probit, European Working Conditions Survey

\begin{abstract}
The purpose of this paper is to compare the extent and determinants of employees' job satisfaction on a European level. The underlying data originate from the European Working Conditions Survey (EWCS) 2005 covering 31 European countries. Beside detailed information about type of work and working conditions the data account for personal and household characteristics including objective and subjective income measures. The result from Ordered-probit regressions for all 31 countries as well as separate regressions for five groups of countries (clustered with respect to level of welfare and type of welfare state) indicate a diversified pattern of explanation of job satisfaction. For all analyzed countries except for countries in Scandinavia and continental Europe the employees' subjective evaluation of income appears to have the strongest effect on job satisfaction. Considering the conflict of working hours with private life, the negative effect on job satisfaction is less distinctive in countries with a lower welfare level. Job security is found to have a stronger impact for UK and Ireland, whereas work autonomy is only found to be among the top ten influences for high level welfare states (UK, Ireland, continental Europe, and Scandinavia).
\end{abstract}

JEL: J28, J81

Keywords: job satisfaction, cross country analysis, working conditions, ordered-probit, European Working Conditions Survey 


\section{Einführung}

Die zentrale in dieser Studie verfolgte Frage lautet: Wirken sich arbeitnehmer- oder arbeitgeberfreundliche flexible Organisationsformen der Arbeit kultur- und wohlstandsniveauunabhängig in gleicher Weise auf die subjektiv wahrgenommene Arbeitszufriedenheit aus? Diese Studie erweitert die bestehende Literatur durch die Analyse einer in einheitlichem Design erhobenen und damit international vergleichbaren Datenbasis, dem European Working Conditions Survey (EWCS). Das EWCS zeichnet sich dadurch aus, dass es neben detaillierten Informationen über Art und Ausgestaltung der Arbeit auch sozioökonomische Informationen für Beschäftigte umfasst. Mit dem EWCS werden dabei Daten analysiert, die mit 31 europäischen Staaten ein breites Spektrum unterschiedlich ausgeprägter Wohlfahrtsstaaten, Wohlfahrtsniveaus und Arbeitsbedingungen abdecken.

Ein Verständnis dessen, wie Personen ihre Arbeitsplatzsituation bewerten, ob sie zufrieden sind oder nicht, ist aus vielerlei Sicht von Interesse. Aus betriebswirtschaftlicher Sicht wurde z.B. eine eingehende Diskussion darüber geführt, ob ein Zusammenhang von Arbeitszufriedenheit und Produktivität besteht (z.B. Pfeffer und Langton 1993, Koys 2001, Patterson et al. 1997, 2004). Ein weiterer Grund für die Bedeutung von Zufriedenheitsanalysen ist, dass diese in den Medien große Aufmerksamkeit erzeugen. Kristensen und Johansson (2008) schließen daraus, dass diese Analysen dadurch mit höherer Wahrscheinlichkeit Politiker zu einer Reaktion veranlassen.

Ökonomen haben sich - neben der nutzenmaximierenden Allokationsanalyse - lange Zeit kaum mit subjektiven Zufriedenheitsangaben beschäftigt. Easterlin (1974) war einer unter den ersten Ökonomen, die sich mit der Analyse subjektiver Merkmale beschäftigt haben und hat den Zusammenhang von Zufriedenheit (life satisfaction) und Einkommen untersucht. Freeman (1978) sowie Akerlof et al. (1988) haben die Bedeutung von Arbeitszufriedenheit untersucht und mit Daten für die USA gezeigt, dass ein Zusammenhang zwischen der angegebenen Arbeitszufriedenheit und dem tatsächlichem Verhalten auf dem Arbeitsmarkt besteht: Niedrigere Arbeitszufriedenheit führt zu einer erhöhten Arbeitsplatzmobilität. Zu dem gleichen Ergebnis kommt Clark (2001) für Großbritannien.

In der Folge hat die Analyse subjektiver Wohlfahrtskennzahlen stark an Bedeutung gewonnen. Dies wurde nicht zuletzt durch die Berufung der „Stiglitz-Sen-Fitoussi-Komission“ durch den französischen Präsidenten Nicolas Sarkozy deutlich. In ihrem Abschlussbericht empfiehlt die Kommission (vgl. Stiglitz et al. 2009) neben objektiven Maßzahlen verstärkt subjektive Kennzahlen zur Wohlfahrtsmessung heranzuziehen. Der Arbeit wird dabei eine wichtige Rolle zugeschrieben, da sie identitätsstiftend sein kann und zudem soziale Partizipation ermöglicht. Wenn Arbeit für die Lebensqualität eine wichtige Rolle spielt, so ist ein genaues Verständnis darüber, welche Faktoren die Arbeitszufriedenheit beeinflussen, von besonderer Bedeutung.

Einfache mikroökonomische Modelle gehen davon aus, dass Individuen Nutzen aus Freizeit und Konsum ziehen. Dementsprechend waren in ökonomischen Studien der Lohn und die Arbeitszeit die beherrschenden Themen bei der Analyse der Arbeitszufriedenheit. Clark (2005) hat mit Daten aus dem International Social Survey Programme ${ }^{2}$ des Jahres 1997 für 19 OECD Staaten untersucht, welche Charakteristika einer Arbeitsstelle von den Arbeitenden als

\footnotetext{
${ }^{2}$ Weiterführende Informationen zu den vom International Social Survey Programme (ISSP) erhobenen Daten finden sich unter www.issp.org.
} 
wichtig eingestuft wurden. Es zeigte sich, - im Widerspruch zu klassischen ökonomischen Modellen - dass flexible Arbeitszeiten und Lohn von den Befragten am seltensten als wichtige Eigenschaften angegeben wurden. Am häufigsten genannt wurden die Arbeitsplatzsicherheit und „eine interessante Tätigkeit“. Ein weiteres Ergebnis war, dass Arbeitszeiten insbesondere dann eine Rolle spielen, wenn der gewünschte Arbeitszeitumfang nicht mit den tatsächlichen Arbeitszeiten in Einklang ist.

Bei der Bewertung der Ergebnisse oben genannter Studie gilt es jedoch zu berücksichtigen, dass die Formulierung in den Fragebögen der genutzten ISSP-Daten lautete „Wie wichtig ist Ihnen eine Stelle, bei der man die Arbeitszeiten oder Arbeitstage selbst festlegen kann?". Diese Formulierung führt dazu, dass die Befragten tendenziell nur positive Aspekte der Arbeitszeitflexibilität bei der Beurteilung in Betracht ziehen. Eine Fragestellung, die nicht nur auf positive Aspekte der Flexibilität abzielt (z.B. ,..., bei der man nicht nachts oder am Wochenende arbeiten muss"), könnte die Ergebnisse deutlich verändern. Arbeit ermöglicht nicht nur soziale Partizipation, sondern kann (z.B. durch regelmäßige Nachtarbeit oder Arbeit an Wochenenden) auch die Teilhabe am gesellschaftlichen Leben erschweren oder verhindern.

Ein weiterer Ansatz der individuellen Wohlfahrtsanalyse des Arbeitsmarkts setzt bei der Übereinstimmung von tatsächlichem und gewünschtem Arbeitszeitumfang an. Merz und Lang (1999) untersuchen dazu - auch speziell für Freie Berufe - mit Längsschnittdaten der Jahre 1985 bis 1994 aus dem Sozio-oekonomischen Panel (SOEP) Determinanten und die Entwicklung der Abweichung der Arbeitszeitpräferenz von den tatsächlichen Arbeitszeiten über einen Zeitraum von zehn Jahren. Merz und Lang kommen dabei zu dem Ergebnis, dass es Freiberuflern durch eine höhere Zeitsouveränität besser gelingt, gewünschte und tatsächliche Arbeitszeiten in Einklang zu bringen. Die Fragestellung, welche Personengruppen besonders von Arbeitszeiten außerhalb der Normalarbeitszeit oder fragmentierten Arbeitszeiten betroffen sind, untersuchen Merz und Burgert (2003, 2004a, 2004b). Einkommenseffekte dieser atypischen Arbeitszeitmuster werden in Merz und Böhm $(2005,2008)$ sowie Merz et al. (2009) analysiert. Dabei zeigen sich signifikante Einkommenseffekte durch Lage und Fragmentierung der Arbeitszeiten. Wie sich flexible Arbeitszeitformen in Deutschland auf die Arbeitszufriedenheit auswirken untersucht Hanglberger (2010) mit Paneldaten aus dem SOEP.

Ziel dieses Beitrags ist es, Niveaus und Bestimmungsfaktoren der Arbeitszufriedenheit von abhängig Beschäftigten auf europäischer Ebene vergleichend zu analysieren. Dieser Vergleich wird durch die Auswertung des EWCS ermöglicht. Das EWCS umfasst neben Großbritannien und Irland, den skandinavischen, kontinental- und südeuropäischen Staaten auch zehn osteuropäische Länder und die Türkei. Dadurch kann eine Analyse divergierender Erklärungsmuster für Länder mit deutlich unterschiedlich ausgeprägten Wohlfahrtsniveaus, Arbeitsbedingungen und Wohlfahrtsstaaten vorgenommen werden.

Um erste Erkenntnisse über die Bestimmungsfaktoren der Arbeitszufriedenheit zu gewinnen, werden deskriptive Analysen auf Länderebene durchgeführt. Für eine genauere multivariate Analyse auf Mikroebene werden Staaten mit ähnlich gestalteten Wohlfahrtsstaaten und Arbeitsmarktcharakteristika in sechs Ländercluster zusammengefasst. Ordered-Probit Regressionsmodelle werden dann zunächst gemeinsam für alle Staaten und in einem zweiten Schritt getrennt nach Ländergruppen spezifiziert, um Unterschiede in den Erklärungsmustern erkennbar zu machen.

Um flexible Arbeitszeitformen in ihren Auswirkungen auf die Arbeitszufriedenheit adäquat berücksichtigen zu können, wird die Ausgestaltung der Arbeitszeiten weitgehend präzise erfasst und deren Auswirkung auf die Arbeitszufriedenheit untersucht. 
Die Arbeiten des Forschungsinstituts Freie Berufe (FFB) der Leuphana Universität Lüneburg (www.leuphana.de/ffb) beschäftigen sich umfangreich mit den Themenbereichen Arbeitsmarkt- und Wohlfahrtsanalysen. Dieser Beitrag einer Wohlfahrtsanalyse, hier zur Arbeitszufriedenheit, soll durch die pointierte Betrachtung der Vergleichsgruppe der Freien Berufen, den abhängig Beschäftigten, zur späteren Analyse und zum Vergleich der Situation der Freien Berufe beitragen.

\section{Zum Aufbau der Arbeit}

Kapitel 2 gibt einen Überblick über die existierende Literatur zum internationalen Vergleich der Arbeitszufriedenheit. In Kapitel 3 wird die Datengrundlage der nachfolgenden empirischen Analyse beschrieben. Die zur Auswertung zur Verfügung stehenden Länder werden in Kapitel 4 in Ländergruppen zusammengefasst. Eine deskriptive Analyse der Arbeitszufriedenheit und der Arbeitsbedingungen auf nationaler Ebene erfolgt in Kapitel 5. In Abschnitt 6 werden die Ergebnisse aus einer multivariaten Ordered-Probit Analyse zu den Bestimmungsfaktoren der Arbeitszufriedenheit auch getrennt für die gebildeten Ländergruppen diskutiert. Zusammengefasst werden die Ergebnisse im abschließenden Abschnitt 7.

\section{Literaturüberblick zum internationalen Vergleich der Arbeitszufriedenheit}

Das Interesse am Thema Arbeitszufriedenheit hat eine Vielzahl von Publikationen hervorgebracht. Der folgende Überblick konzentriert sich auf die Literatur, die sich der Erklärung der Arbeitszufriedenheit auf internationaler Ebene widmet. Eine Zusammenfassung der Forschung aus soziologischer und psychologischer Perspektive zum Thema der Arbeitszufriedenheit findet sich z.B. in Warr (1999). Ein Überblick über ökonomische Studien zur Erklärung der Arbeitszufriedenheit und die dabei verwendeten Methoden ist z.B. in D'Addio et al. (2007) zu finden.

Empirische Analysen, die sich mit dem internationalen Vergleich der Bestimmungsfaktoren und Niveaus der Arbeitszufriedenheit beschäftigten, bauen auf der Annahme auf, dass subjektive Zufriedenheitsangaben nicht entscheidend durch kulturelle oder sprachliche Unterschiede beeinflusst werden. Kristensen und Johansson (2008) untersuchen dazu in ihrer Studie, ob Differenzen, die sich in den Arbeitszufriedenheitsniveaus auf Länderebene ergeben haben, darauf zurückgeführt werden können, dass kulturelle Unterschiede in der Wahrnehmung der Bewertungsskala existieren. Dazu wurden Probanden aus sieben europäischen Ländern aufgefordert ihre aktuelle Arbeitszufriedenheit anzugeben und zudem mehrere hypothetische Arbeitsplatzsituationen anhand derselben Skala zu bewerten. Das Ergebnis der Studie von Kristensen und Johansson ist, dass kulturelle Unterschiede auch in westeuropäischen Ländern existieren, deren wirtschaftliche Entwicklung relativ ähnlich ist. Bei Kontrolle für diese kulturellen Bewertungsunterschiede ergibt sich eine teilweise veränderte Länderrangliste der Arbeitszufriedenheit. Es ist jedoch anzumerken, dass diese Abweichungen großteils nicht statistisch signifikant sind.

Layard (2003) weist für die internationale Vergleichbarkeit von Zufriedenheitsangaben darauf hin, dass sich Probleme durch abweichende Wortbedeutungen in verschiedenen Sprachen ergeben können. Eine empirische Überprüfung dieser Vermutung durch Veenhoven (2008), bei der Probanden ihre Situation in Bezugnahme auf die drei unterschiedliche Kriterien bewerten, zeigt jedoch, dass nationale Unterschiede in subjektiven Zufriedenheitsangaben dadurch nicht erklärt werden können. Für sprachliche Differenzen im Verständnis der Bewer- 
tungsskala gibt es bislang keine empirische Bestätigung. Im Folgenden wird deshalb davon ausgegangen, dass die Arbeitszufriedenheit international vergleichbar ist.

$\mathrm{Zu}$ den Studien, die internationale Datenbasen nutzen, um Determinanten der Arbeitszufriedenheit zu bestimmen, gehört der Beitrag von Clark (2005). Clark untersucht dort Daten des International Social Survey Programme (ISSP) für 19 Länder. Mit einfachen Befragungen und Ordered-Probit Regressionsmodellen kommt Clark (2005) dabei zu dem Ergebnis, dass die Arbeitsplatzsicherheit und das Interesse an der ausgeübten Tätigkeit den stärksten Einfluss ausüben. Das Einkommen und die Arbeitszeiten spielen demnach eine deutlich geringere Rolle, als dies klassische ökonomische Modelle prognostizieren. Ein Vergleich der Bestimmungsfaktoren in den betrachteten Ländern wird hierbei jedoch nicht vorgenommen. Eine Berücksichtigung länderspezifischer Eigenschaften erfolgt lediglich durch die Integration von Dummyvariablen für jedes Land.

Origo und Pagani (2006) untersuchen den Zusammenhang unterschiedlicher Aspekte der Flexibilität (Tätigkeit, Bezahlung, Befristung, etc.) mit der Arbeitszufriedenheit. Als Datenbasis dazu dient das Special Eurobarometer 56.1 aus dem Jahr 2001 für 15 Länder Europas. Ergebnis der Studie sind Abweichungen in den Determinanten der Arbeitszufriedenheit nach Länderclustern. So wirkt sich Autonomie bei der Arbeit nur in Nordeuropa signifikant auf die Zufriedenheit aus, während der negative Effekt von Teilzeitarbeit und flexiblen Arbeitszeiten besonders in Kontinentaleuropa hoch ist. Eine Schwäche der verwendeten Daten ist, dass das Einkommen der Probanden nicht objektiv erhoben wurde. Da viele Flexibilitätsformen auch mit dem Einkommen korrelieren, ergibt sich hieraus ein erhebliches Omitted-Variable Problem.

Sousa-Poza und Sousa-Poza (2000) analysieren basierend auf Daten des International Social Survey Programme (ISSP) aus dem Jahr 1997 internationale Unterschiede im Niveau und in den Bestimmungsfaktoren der Arbeitszufriedenheit. Neben ost- und westeuropäischen Staaten konnten dabei auch Informationen zu den USA, Neuseeland, Japan und Israel ausgewertet werden. Die Stärke der Datenbasis ist, dass ein weltweiter Vergleich der Arbeitszufriedenheit für 21 Staaten ermöglicht wird. Die Schwäche dieser Daten besteht darin, dass kaum weniger entwickelte Staaten mit aufgenommen wurden, die einen kontraststarken Vergleich der Bestimmungsfaktoren der Arbeitszufriedenheit ermöglichen würden. Des Weiteren enthalten die ISSP Daten keine objektiven Einkommensinformationen und teilweise auch für große Staaten nur relativ kleine Stichproben (z.B. Spanien oder Großbritannien). Nach einem Vergleich durchschnittlicher Arbeitszufriedenheitsniveaus der ISSP-Teilnehmerstaaten schätzen SousaPoza und Sousa-Poza (2000) Ordered-Probit Regressionsmodelle für jedes Land, um den Einfluss einzelner Faktoren auf die Arbeitszufriedenheit international zu vergleichen. Die Autoren kommen zu dem Ergebnis, dass eine interessante Tätigkeit und ein gutes Verhältnis zu den Vorgesetzten länderübergreifend den stärksten Einfluss auf die Arbeitszufriedenheit haben. Für die osteuropäischen Staaten ergab sich zudem ein starker Einfluss der subjektiven Einkommensbewertung. Vor dem Hintergrund der genannten Datenprobleme und sich ergebender Probleme, wenn subjektive Einschätzungen durch andere subjektive Bewertungen derselben Person erklärt werden (vgl. Hamermesh 2004), sind die Ergebnisse der Studie mit Skepsis zu betrachten.

Der gleiche Kritikpunkt gilt auch für eine weitere Studie, die mit Daten für zehn europäische Staaten aus dem European Community Household Panel Survey (ECHP) arbeitet: Nicoletti (2006) verfolgt dabei den Ansatz, die Arbeitszufriedenheit durch die subjektive Zufriedenheiten mit Teilaspekten der Arbeit (z.B. mit dem Einkommen, mit der Art der Tätigkeit, etc.) zu erklären. 
Ahn und García (2004) untersuchen in ihrer Studie mit Daten aus dem European Community Household Panel Survey (ECHP) der Jahre 1994 bis 2001 Bestimmungsfaktoren der globalen Arbeitszufriedenheit und Bereichszufriedenheiten. Im ECHP konnte dazu auf Daten aus acht westeuropäischen Ländern zurückgegriffen werden. Zur Analyse wurden die Daten von 1994 bis 2001 zusammengelegt (,,pooling“) und ein lineares Regressionsmodell aller Jahre geschätzt. Die Arbeitszufriedenheit wird demnach am stärksten durch den Gesundheitszustand beeinflusst. Daneben spielen das Einkommen, der berufliche Status, die Art des Arbeitsvertrags und die Übereinstimmung von beruflicher Tätigkeit und eigener Qualifikation eine entscheidende Rolle. Eine Schwäche dieser Schätzung ist, dass keine relativen Einkommenspositionen berücksichtigt wurden und die Art der Tätigkeit im ECHP verglichen mit der European Working Conditions Survey (EWCS, siehe Abschnitt 3) nur ungenau erfasst ist.

Eine Studie basierend auf Daten aus dem EWCS des Jahres 2000 stammt von Martin (2002). Dabei werden für Selbständige Bestimmungsfaktoren der Arbeitszufriedenheit in 15 europäischen Ländern untersucht. Die Analyse erfolgt dabei für alle Länder gemeinsam ohne auf länderspezifische Unterschiede einzugehen.

Zusammenfassend kann festgestellt werden, dass die bestehende Literatur zum internationalen Vergleich der Bestimmungsfaktoren der Arbeitszufriedenheit zu dem Ergebnis kommt, dass der Arbeitsinhalt und die Art der Tätigkeit entscheidende Faktoren sind. Die Ergebnisse in Bezug auf das Einkommen und die Flexibilität der Arbeitszeiten variieren zum Teil bedingt durch die unterschiedliche Datenqualität. Die Schwäche bestehender Analysen ist insbesondere in den verwendeten Datenbasen zu sehen. Es fehlen entweder präzise Informationen über die Arbeitsbedingungen, oder objektive Einkommensinformationen. Diese Lücke soll in dieser Arbeit durch die Auswertung des EWCS geschlossen bzw. zumindest verkleinert werden.

\section{Datengrundlage - European Working Conditions Survey}

Die European Foundation for the Improvement of Living and Working Conditions (Eurofound) wurde 1975 vom Europäischen Rat als EU-Körperschaft eingerichtet. Aufgabe von Eurofund ist es, für Politik, Arbeitgeber und Gewerkschaften Informationen über den Wandel der Lebens- und Arbeitsbedingungen, der industriellen Beziehungen und der Work-LifeBalance zu sammeln, zur Verfügung zu stellen und zu erforschen. ${ }^{3}$

Im Rahmen dieser Aufgabenstellung wurden mit dem European Working Conditions Survey (EWCS) bis 2010 fünf international einheitliche Befragungen in Europa durchgeführt. ${ }^{4}$ Die Daten der letzten Erhebung im Jahr 2010 standen zum Zeitpunkt der Erstellung dieser Arbeit noch nicht zur Verfügung. Ziel der Erhebung ist, die Arbeitsbedingungen von abhängig Beschäftigten und Selbständigen in Europa in einheitlicher Art und Weise zu erfassen, die Analyse von Zusammenhängen und Auswirkungen der Arbeitsbedingungen zu ermöglichen und aktuelle Entwicklungen der Arbeitsbedingungen zu beobachten. ${ }^{5}$ Im Jahr 2005 wurden über 100 Items mit Informationen über die Erwerbstätigkeit, die Organisation und Dauer der Ar-

3 Weiterführende Informationen zur Organisation und Aufgabenstellung von Eurofund finden sich auf der Internetseite www.eurofound.europa.eu.

4 Erhebungen fanden mit im Zeitverlauf zunehmendem Themenspektrum und Teilnehmerländern in den Jahren 1990/91 (EC12), 1995/96 (EU15), 2001 (EU15), 2005 (EU 25 plus Bulgarien, Rumänien, Norwegen, Türkei, Schweiz) und 2010 (EU27, Norwegen, Kroatien, Mazedonien, Türkei, Albanien, Montenegro und Kosovo) statt.

5 Siehe hierzu und für weiterführende Informationen auch die EWCS Internetseite www.eurofound.europa.eu/ewco/surveys. 
beitszeiten, die Organisation der Arbeit, Fortbildungsmaßnahmen, Risikofaktoren für Sicherheit und Gesundheit, das Ausmaß an Mitbestimmung, Einkommen und finanzielle Sicherheit sowie Gesundheit und die Zufriedenheit mit den Arbeitsbedingungen abgefragt (ParentThirion et al. 2007).

Die in dieser Arbeit ausgewerteten Daten sind der vierten Befragungswelle entnommen. Für diese wurden von 19. September bis 30. November 2005 in Zypern, Estland, Finnland, Luxemburg, Malta und Slowenien jeweils ca. 600 Personen befragt. In allen anderen Ländern wurden ca. 1.000 Personen befragt. Insgesamt ergibt sich so ein Datensatz mit Informationen über 29.200 Probanden. Erhoben wurden Informationen von Personen ab einem Alter von 15 Jahren (für Spanien, Norwegen und Großbritannien galt abweichend ein Mindestalter von 16 Jahren), die in der Referenzwoche erwerbstätig waren. Für die Erwerbstätigkeit galt dabei die Eurostat Abgrenzung. Danach gilt als erwerbstätig, wer in der Referenzwoche mindestens eine Stunde gegen Bezahlung gearbeitet hat.

Die Befragungen wurden als face-to-face Interviews in der Wohnung der Probanden durchgeführt. Die Stichprobenziehung erfolgte nach Regionen und Urbanisierungsgrad geschichtet. Die kontaktierten Haushalte wurden nach einem Random-Walk-Verfahren ausgewählt und bis zu viermal kontaktiert, wenn ein Kontaktversuch erfolglos war. Abweichungen von diesem Verfahren gab es für Norwegen, die Schweiz und Schweden. Hier wurden die Personen basierend auf einem Telefonregister ausgewählt und die Befragungen telefonisch durchgeführt. In Haushalten mit mehreren Erwerbstätigen wurde immer die Person zur Befragung ausgewählt, deren Geburtstag als letztes vor dem Befragungszeitpunkt war.

Eine ausführliche Beschreibung der Erhebungsmethoden, Rücklaufquoten und weiterer Datensatzmerkmale findet sich für die vierte Welle in Parent-Thirion et al. (2007). Für alle weiteren Wellen werden auf der Internetseite www.eurofound.europa.eu/ewco/surveys die entsprechenden Informationen zur Verfügung gestellt. Ebenso sind dort die Fragebögen und Showcards, die bei der Erhebung zum Einsatz kamen, einsehbar.

Die Arbeitszufriedenheit wurde im EWCS auf einer Viererskala erhoben. Die Probanden konnten die Aussage „Alles in allem gesehen: Sind Sie mit den Arbeitsbedingungen in Ihrem Hauptberuf..." mit den vier Antwortkategorien „,sehr zufrieden“, ,zufrieden“, „,nicht sehr zufrieden“ oder ,überhaupt nicht zufrieden“ vervollständigen. ${ }^{6}$ Wenn im Folgenden von der Arbeitszufriedenheit die Rede ist, dann wird auf diese Frage Bezug genommen.

Des Weiteren wurden aus dem EWCS für die folgenden Analysen Informationen zur Person und Haushaltssituation, über das Arbeitsverhältnis, die Arbeitszeiten, über die Art der Tätigkeit, das Einkommen, die Aufstiegsmöglichkeiten und persönliche Beziehungen am Arbeitsplatz genutzt. Detaillierte Informationen zur Operationalisierung finden sich im Anhang in Tabelle 10 .

Die Daten aus dem EWCS haben im Vergleich zu anderen Datenbasen entscheidende Vorteile. Im Vergleich zu Daten aus dem ISSP oder dem Special Eurobarometer 2001 werden objektive Einkommensinformationen erhoben. Das Einkommen wurde im EWCS in zehn Gruppen, die sich jeweils an den nationalen Einkommensdezilen orientierten, erhoben. Somit ermöglichen die Daten eine Berücksichtigung der absoluten wie auch der relativen Einkommensposition. Für die Analyse der Arbeitszufriedenheit, bei der anzunehmen ist, dass das

6 Die englische Formulierung im Fragebogen lautet: "On the whole, are you very satisfied, satisfied, not very satisfied or not at all satisfied with working conditions in your main paid job?" und konnte mit der Skala "Very satisfied", "Satisfied", "Not very satisfied" "Not at all satisfied" bewertet werden. 
Einkommen einen Einfluss ausübt, ist dies von besonderer Bedeutung. Im Vergleich mit dem ECHP enthält das EWCS eine deutlich größere Anzahl an Ländern mit größeren wirtschaftlichen und kulturellen Unterschieden. Zudem wird das EWCS zielgerichtet zur Messung der Qualität der Lebens- und Arbeitsbedingungen erhoben. In Hinsicht auf die Genauigkeit der Erhebung der Arbeitsplatzmerkmale ist die Qualität der EWCS-Daten deshalb besonders hervorzuheben.

\section{Länderklassifikation}

Um die empirischen Analysen bei einem Vergleich von 31 Ländern übersichtlich zu gestalten, wird eine Klassifikation der Staaten in Anlehnung an bestehende Klassifikationen nach Wohlfahrtsregimes vorgenommen.

Eine der einflussreichsten Arbeiten zur Klassifikation von Wohlfahrtsregimes stammt von Esping-Andersen (1990). Der Autor ordnet dabei Staaten nach dem Level der Dekommodifizierung. Unter Dekommodifizierung wird ,the degree to which individuals or families can uphold a socially acceptable standard of living independently of market participation" (Esping-Andersen 1990: 47) verstanden. Bestimmt wird der Grad der Dekommodifizierung durch die Bedingungen, die eine Person erfüllen muss, um Anspruch auf Sozialleistungen zu haben, den anspruchsberechtigten Personenkreis und die Höhe der Sozialleistungen. Ein hohes Maß an Dekommodifizierung liegt demnach dann vor, wenn keine Anspruchsvoraussetzungen (z.B. vorherige Beitragszahlungen in eine Sozialversicherung) erfüllt werden müssen und die Höhe der Leistungen ausreicht, um einen angemessenen Lebensstandard zu halten. Es werden also mehrere Eigenschaften des Wohlfahrtsregimes in einer Dimension zusammengefasst.

Tabelle 1: Klassifikation Wohlfahrtsregime

\begin{tabular}{ll}
\hline Esping-Andersen & Bonoli+2 \\
\hline Liberal: Großbritannien, Schweiz & Englischsprachig: UK, Irland \\
Konservativ: Deutschland, Frankreich, & Kontinentaleuropäisch: Niederlande, Frankreich, \\
Italien, Irland, Belgien, Österreich & Belgien, Deutschland, Luxemburg, Österreich \\
Sozial-demokratisch: Finnland, Nieder-- Skandinavisch: Schweden, Finnland, Norwegen, Dä- \\
lande, Norwegen, Dänemark, Schweden & nemark \\
& Südeuropäisch: Italien, Spanien, Griechenland, \\
& Schweiz, Portugal, Kroatien, Malta, Cypern \\
& Osteuropäisch: Tschechien, Estland, Lettland, Litau- \\
& en, Polen, Slowakei, Slowenien, Bulgarien, Rumänien, \\
& Ungarn \\
& Türkei \\
&
\end{tabular}

Quelle: Eigene Zusammenstellung.

Ein Überblick der Klassifikation nach Esping-Andersen (1990) findet sich in Tabelle 1. Bonoli (1997) kritisiert dieses Modell, da es nicht zwischen sozialversicherungs- und steuerfinanzierten Modellen ${ }^{7}$ unterscheidet. Dies führt dazu, dass z.B. die Niederlande, wo Ansprüche zu großen Teilen auf vorangegangenen Beitragszahlungen in die Sozialversicherung beruhen, in die gleiche Gruppe eingeordnet werden, wie Dänemark, ein steuerfinanzierter Sozialstaat.

7 Überwiegend steuerfinanzierte Systeme werden in der Literatur häufig auch als Beveridge, beitragsfinanzierte als Bismarck Wohlfahrtsstaaten bezeichnet. 
Weiter kritisiert Bonoli (1997), dass das Modell nicht geeignet ist, um insbesondere in Südeuropa Staaten adäquat zu erfassen, in denen familiäre und andere informelle Netzwerke eine bedeutende Rolle spielen.

\section{Abbildung 1: Bonoli Klassifikation Wohlfahrtsstaaten}

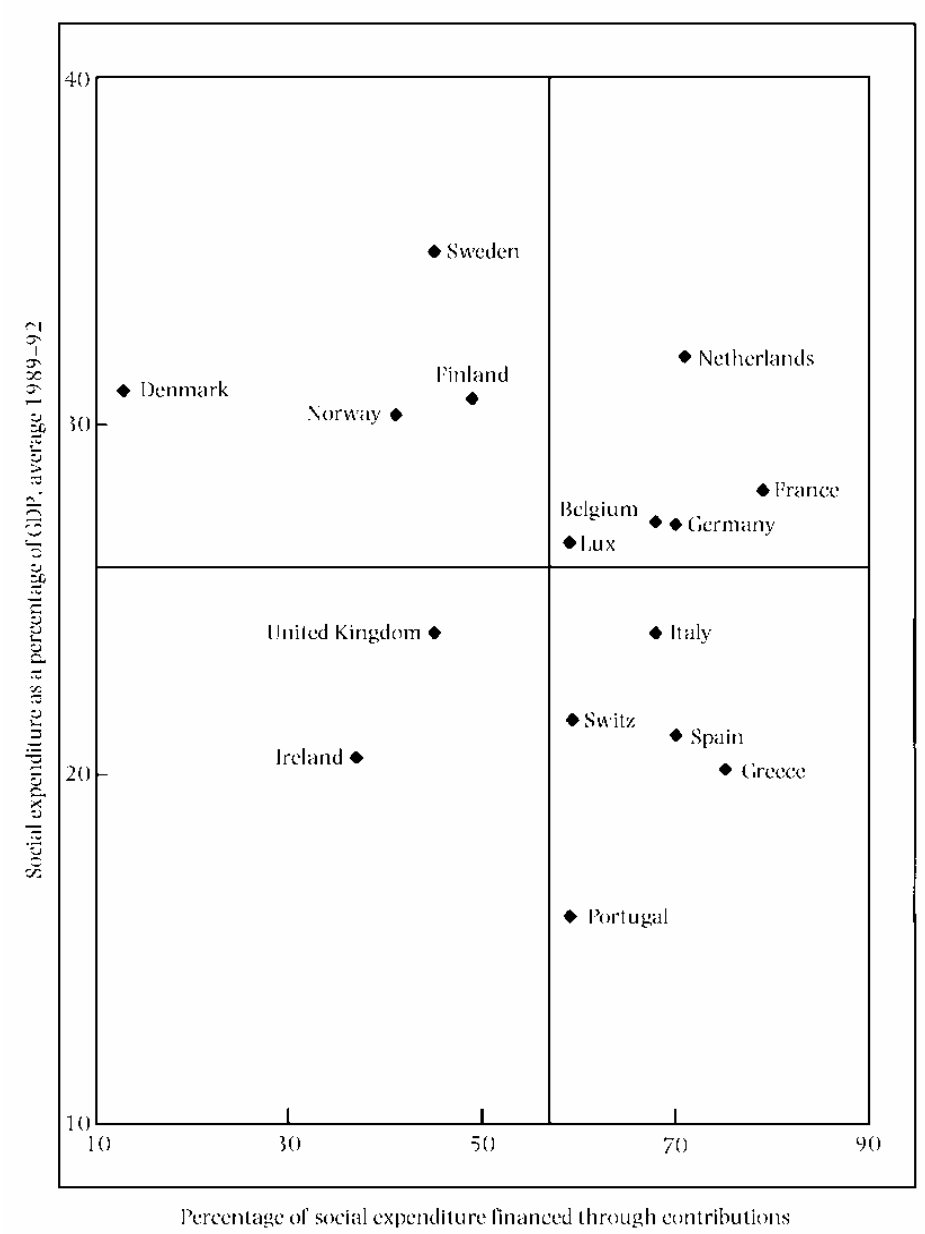

Quelle: Bonoli (1997: 361).

Um die Schwächen der Klassifikation nach Esping-Andersen (1990) zu umgehen, wählt Bonoli (1997) einen zweidimensionalen Ansatz. Eine Dimension misst dabei die Höhe der Sozialleistungen als Anteil der Sozialausgaben am Bruttoinlandsprodukt. Eine zweite Dimension erfasst, ob es sich um ein steuerfinanziertes (Beveridge) oder beitragsfinanziertes (Bismarck) System handelt. Als Indikator wird dabei der Anteil der beitragsfinanzierten Sozialausgaben an allen Sozialausgaben verwendet. Ein guter Überblick zur Abgrenzung von Beveridge und Bismarck Modellen findet sich in Bonoli (1997: 357). Mit Hilfe dieser beiden Dimensionen werden vier Typen von Wohlfahrtsstaaten unterschieden (siehe Abbildung 1). Als erste Gruppe lassen sich die skandinavischen Staaten zusammenfassen. Sie zeichnen sich durch ein hohes Niveau an Sozialleistungen bei überwiegender Steuerfinanzierung aus. Die englischsprachigen Länder Großbritannien und Irland stellen das zweite Cluster dar. Sie verfügen ebenfalls über eine überwiegende Steuerfinanzierung allerdings bei einem niedrigeren Niveau an Sozialausgaben. Die beiden verbleibenden Cluster kontinentaleuropäischer und südeuropäischer Staaten sind gekennzeichnet durch eine starke Beitragsfinanzierung der Sozialausgaben, 
wobei das Niveau der Sozialausgaben in den kontinentaleuropäischen Staaten deutlich höher liegt.

Im Folgenden wird sich dieser Klassifikation der Wohlfahrtsstaaten angeschlossen. Länder für die Bonoli keine Zuordnung vorgenommen hat, wurden dementsprechend ergänzt. Österreich wurde der Gruppe kontinentaleuropäischer Staaten zugeordnet, Kroatien, Malta und Zypern den südeuropäischen Staaten. Für die Gruppe postkommunistischer Staaten in Osteuropa wurde ein eigenes Cluster gebildet. Für die Türkei, die im Rahmen dieser Arbeit ebenfalls analysiert wird, wurde soweit möglich keine Gruppenzuordnung vorgenommen, da sich die Türkei in Hinblick auf die Arbeitsbedingungen stark von den anderen betrachteten Ländern unterscheidet (vgl. Tabelle 2). Insgesamt ergeben sich damit sechs Ländercluster (Skandinavien, Großbritannien und Irland, Kontinentaleuropa, Südeuropa, Osteuropa, Türkei), deren Bildung sich an Bonoli (1997) orientiert. Diese Klassifikation wird im weiteren Verlauf als Bonoli +2 bezeichnet und findet sich überblicksartig in Tabelle 1.

Eine Zusammenfassung mehrerer Staaten in Gruppen ist immer schwierig und kann letztlich nie die Vielzahl kultureller Differenzen und die Fülle der Eigenschaften einzelner Wohlfahrtsregime erfassen. Im Sinne der Darstellbarkeit der empirischen Analysen erscheint sie dennoch notwendig. Für eine ausführliche Diskussion zur Klassifikation von Wohlfahrtsregimes siehe neben Esping-Andersen (1990) und Bonoli (1997) auch Arts und Gelissen (2002), Ferrera (1996) und Sharkh und Gough (2010). Eine genauere Betrachtung und Untergliederung der hier als osteuropäisch zusammengefassten Staaten findet sich in Fenger (2007).

\section{Arbeitszufriedenheit in Europa - Ein deskriptiver Vergleich}

Ein erster deskriptiver Vergleich der Arbeitszufriedenheit (im EWCS gemessen auf einer Skala von 1 ,,überhaupt nicht zufrieden“ bis 4 ,sehr zufrieden“) in Europa zeigt, dass das durchschnittliche Niveau der Zufriedenheit in den meisten Ländern hoch ist. Ein Ergebnis das in Übereinstimmung mit vergleichbaren Studien ist (z.B. Sousa-Poza und Sousa-Poza 2000). Es lässt sich aber ebenfalls erkennen, dass das Niveau der Arbeitszufriedenheit auch auf aggregierter nationaler Ebene stark variiert (vgl. Abbildung 2). Während Großbritannien, Irland und die skandinavischen Staaten ein sehr hohes Niveau an Arbeitszufriedenheit aufweisen, finden sich auf niedrigerem Niveau viele osteuropäische Staaten. Besonders deutlich fällt die Türkei im Vergleich mit den anderen 30 EWCS-Teilnehmerstaaten ab. Insbesondere unter Berücksichtigung des meist positiv ausfallenden Antwortverhaltens auf Zufriedenheitsfragen ist die durchschnittliche Arbeitszufriedenheit von rund 2,5 auffallend. Während der Anteil derjenigen, die angeben mit ihren Arbeitsbedingungen nicht so sehr oder überhaupt nicht zufrieden zu sein, in Dänemark bei unter sieben Prozent und in Deutschland bei knapp elf Prozent liegt, beträgt dieser Anteil in Griechenland 39 Prozent und in der Türkei sogar annähernd 46 Prozent (vgl. Tabelle 8 im Anhang). Im europäischen Vergleich liegt Deutschland noch im oberen Drittel. Angeführt wird die Rangliste, wie dies auch in anderen Studien (vgl. Blanchflower und Oswald 1999, Sousa-Poza und Sousa-Poza 2000, Clark 2005, Nicoletti 2006, Eurobarometer 2009) der Fall ist, von Dänemark.

Aus Abbildung 3 wird ersichtlich, dass sich auch die im vorangegangenen Abschnitt gebildeten Ländergruppen im Niveau der Arbeitszufriedenheit deutlich unterscheiden. Hier wird die Rangliste von Großbritannien und Irland angeführt, gefolgt von den skandinavischen Staaten, Kontinentaleuropa und Südeuropa. Am Ende der Rangliste finden sich die osteuropäischen Staaten sowie Griechenland und die Türkei. 


\section{Abbildung 2: Durchschnittliche Arbeitszufriedenheit der 31 EWCS Länder im Ver- gleich}

(1=überhaupt nicht zufrieden, 4=sehr zufrieden)

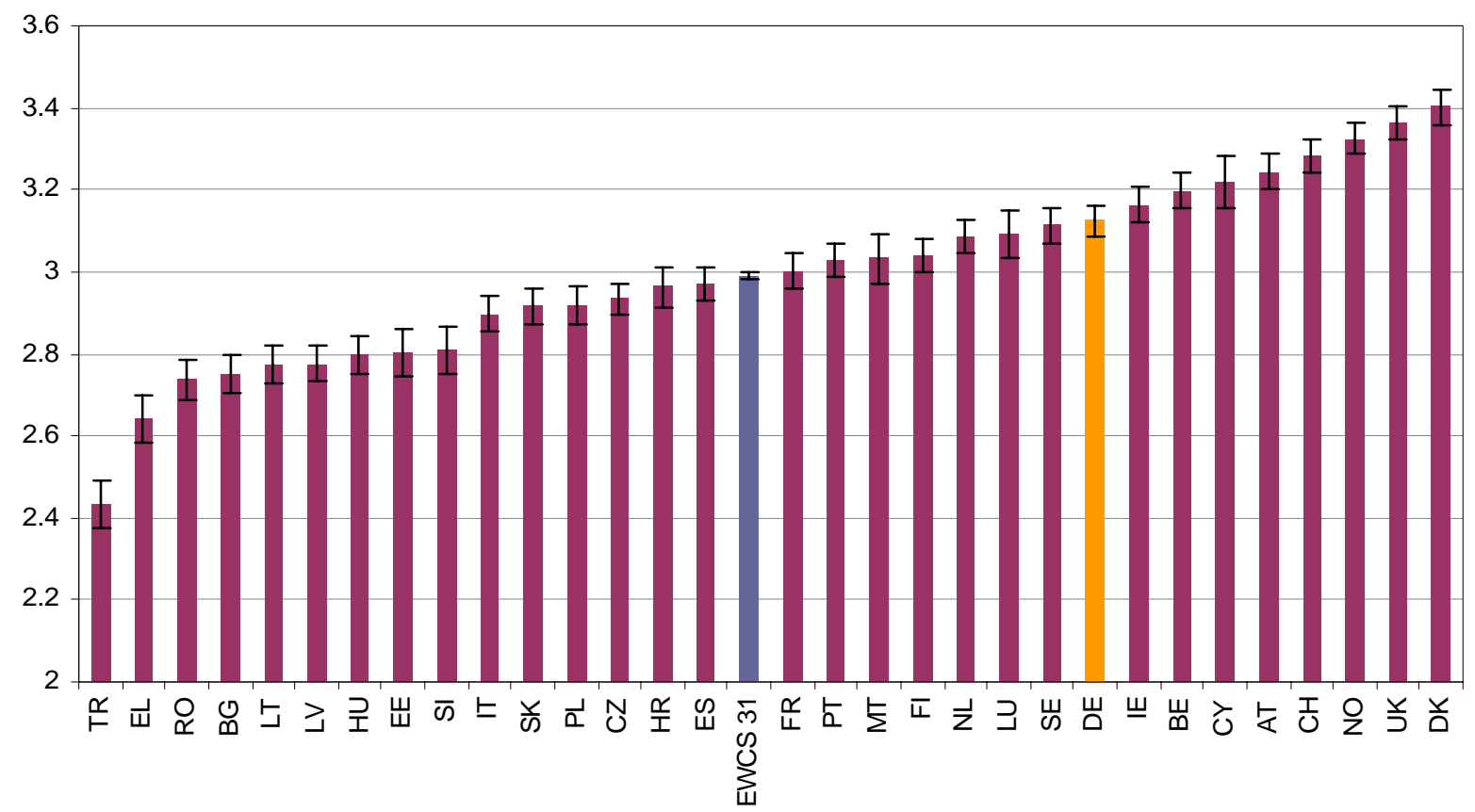

Länderkürzel: AT Österreich, BE Belgien, BG Bulgarien,. CY Zypern, CZ Tschechien, DK Dänemark, EE Estland, FI Finnland, FR Frankreich, DE Deutschland, EL Griechenland, HU Ungarn, IE Irland, IT Italien, LV Lettland, LT Litauen, LU Luxemburg, MT Malta, NL Niederlande, PL Polen, PT Portugal, RO Rumänien, SK Slowakei, SI Slowenien, ES Spanien, SE Schweden, UK Großbritannien, HR Kroatien, NO Norwegen, CH Schweiz, TR Türkei.

Quelle: Eigene Berechnungen basierend auf EWCS 2005; gewichtet; 95 \% Konfidenzintervalle.

Um mögliche Erklärungen für Unterschiede in der beobachteten Arbeitszufriedenheit zu finden, werden in Tabelle 2 Faktoren, die einen Einfluss auf die Arbeitszufriedenheit ausüben können, auf nationaler Ebene verglichen. Dabei zeigen sich erhebliche Unterschiede in den Arbeitsbedingungen. Die durchschnittlichen Einkommen betreffend heben sich Großbritannien, Irland und die skandinavischen Staaten vom Rest ab. Die niedrigsten Durchschnittseinkommen entfallen auf die osteuropäischen Staaten und die Türkei. Dies spricht für eine wichtige Rolle des Einkommens auf die Arbeitszufriedenheit. Bei der Befristung der Arbeitsverhältnisse ist das Bild weniger eindeutig. Während die Gruppe kontinental-, osteuropäischer und skandinavischer Staaten über einen hohen Anteil an unbefristeten Beschäftigungen verfügen, ist dieser in Irland, Großbritannien, Südeuropa und der Türkei relativ niedrig. Dabei ist anzumerken, dass eine unbefristete Beschäftigung nicht immer über Ländergrenzen hinweg vergleichbar ist. Eine unbefristete Beschäftigung kann durch einen umfangreichen Kündigungsschutz (wie z.B. in Deutschland) hohe Beschäftigungssicherheit für den einzelnen bieten, in Ländern mit weniger ausgeprägtem Kündigungsschutz, wie z.B. Dänemark, ist dies jedoch nicht der Fall (vgl. Rhein 2010).

Deutliche Indizien sprechen darüber hinaus für einen starken Einfluss des zeitlichen Arbeitsumfangs auf die Arbeitszufriedenheit. Hier fällt auf, dass Arbeitnehmer in Länderclustern mit niedriger Arbeitszufriedenheit deutlich mehr Wochenarbeitsstunden aufweisen. Auch das Niveau der körperlichen Belastung ist in Süd- und Osteuropa sowie der Türkei deutlich höher. In Bezug auf das Niveau flexibler Arbeitszeitgestaltung fällt auf, dass die skandinavischen Staaten nicht nur ein hohes Niveau an arbeitnehmerfreundlicher Flexibilität sondern auch ar- 
beitgeberfreundlicher Flexibilität aufweisen. Auch in Bezug auf Autonomie in der Arbeitsgestaltung und anspruchsvolle Tätigkeiten heben sich die skandinavischen Staaten vom Rest der untersuchten Länder ab.

\section{Abbildung 3: Durchschnittliche Arbeitszufriedenheit der Bonoli+2 Cluster}

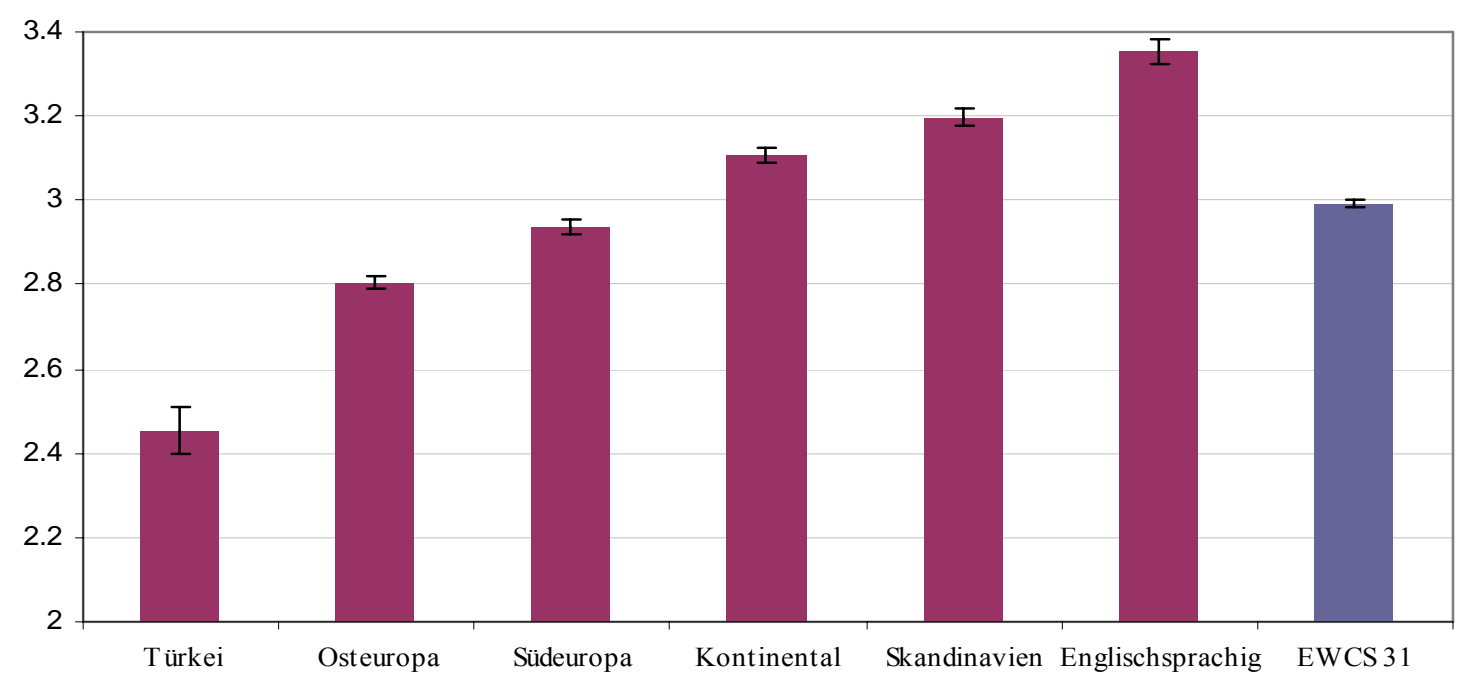

Quelle: Eigene Berechnungen basierend auf EWCS 2005; gewichtet; 95 \% Konfidenzintervalle der Mittelwerte; $1=$ überhaupt nicht zufrieden, $4=$ sehr zufrieden.

Zur Erklärung der auffallend niedrigen Arbeitszufriedenheit in der Türkei fallen mehrere Aspekte auf. So verfügt die Türkei über den niedrigsten Anteil unbefristet Beschäftigter, über die deutlich höchste Anzahl an Wochenarbeitsstunden, das höchste Niveau an atypischen Arbeitszeiten und die Einschätzung, eine sinnvolle und anspruchsvolle Tätigkeit auszuüben, ist im Vergleich zu den anderen Staaten geringer ausgeprägt.

Um Zusammenhänge der Arbeitszufriedenheit auf nationaler Ebene mit den länderspezifischen Arbeitsmarktbedingungen in einem ersten Schritt auf bivariater Ebene zu identifizieren und quantifizieren, gibt Tabelle 3 die entsprechenden Korrelationskoeffizienten wieder. ${ }^{8}$ Dabei zeigen sich die stärksten Korrelationen der Arbeitszufriedenheit mit der Wochenarbeitszeit, der körperlichen Belastung und dem Einkommen. Geringere aber ebenfalls signifikante Korrelationen ergeben sich für den Inhalt der Tätigkeit und die persönlichen Beziehungen am Arbeitsplatz. Grundsätzlich erscheint dieses erste Ergebnis plausibel und steht im Widerspruch zu einer Vielzahl von Querschnittsstudien auf Mirkoebene (vgl. z.B. Sousa-Poza und Sousa-Poza 2000, Clark 2005), die den Arbeitszeiten und dem Einkommen eine untergeordnete Rolle bei der Bestimmung der Arbeitszufriedenheit zuweisen.

\footnotetext{
${ }^{8}$ Eine ausführliche Analyse von Bestimmungsfaktoren subjektiver Wohlfahrtsindikatoren auf Makroebene findet sich in Diener et al. (1995).
} 
Tabelle 2: Durchschnittliche Arbeitsmarktcharakteristika der 31 EWCS Länder 2005

\begin{tabular}{|c|c|c|c|c|c|c|c|c|c|c|c|c|}
\hline Land & $\begin{array}{c}\text { Arbeits- } \\
\text { zufriedenheit }\end{array}$ & Einkommen & Unbefristet & $\begin{array}{l}\text { Arbeits- } \\
\text { stunden } \\
\text { /Woche }\end{array}$ & $\begin{array}{c}\text { Körperliche } \\
\text { Belastung }\end{array}$ & $\begin{array}{c}\text { Ungünstige } \\
\text { Arbeitszeiten }\end{array}$ & $\begin{array}{c}\text { Flexibilität } \\
\text { für } \\
\text { Arbeitgeber }\end{array}$ & $\begin{array}{c}\text { Flexibilität } \\
\text { für } \\
\text { Arbeitnehmer } \\
\end{array}$ & Autonomie & Sinnvoll & $\begin{array}{c}\text { Persönliche } \\
\text { Beziehungen }\end{array}$ & $\begin{array}{c}\text { Anspruchs- } \\
\text { voll }\end{array}$ \\
\hline Irland & 3.15 & 2089 & 0.58 & 37.83 & 0.13 & 0.34 & 0.25 & 0.52 & 0.72 & 0.94 & 0.87 & 0.59 \\
\hline Großbritannien & 3.37 & 1880 & 0.69 & 35.42 & 0.13 & 0.32 & 0.26 & 0.49 & 0.66 & 0.93 & 0.86 & 0.58 \\
\hline Belgien & 3.21 & 1405 & 0.90 & 37.21 & 0.15 & 0.32 & 0.28 & 0.48 & 0.74 & 0.94 & 0.76 & 0.59 \\
\hline Deutschland & 3.14 & 1494 & 0.86 & 37.77 & 0.20 & 0.30 & 0.29 & 0.31 & 0.62 & 0.93 & 0.78 & 0.62 \\
\hline Frankreich & 3.04 & 1480 & 0.86 & 35.54 & 0.19 & 0.27 & 0.24 & 0.45 & 0.69 & 0.95 & 0.71 & 0.55 \\
\hline Luxemburg & 3.09 & 2480 & 0.90 & 38.28 & 0.15 & 0.25 & 0.22 & 0.50 & 0.74 & 0.96 & 0.78 & 0.58 \\
\hline Niederlande & 3.09 & 1640 & 0.80 & 33.41 & 0.14 & 0.31 & 0.32 & 0.61 & 0.76 & 0.96 & 0.79 & 0.72 \\
\hline Österreich & 3.26 & 1348 & 0.82 & 39.82 & 0.16 & 0.31 & 0.30 & 0.49 & 0.66 & 0.96 & 0.79 & 0.68 \\
\hline Dänemark & 3.39 & 2035 & 0.81 & 36.77 & 0.15 & 0.28 & 0.35 & 0.59 & 0.82 & 0.98 & 0.88 & 0.77 \\
\hline Finnland & 3.04 & 1517 & 0.82 & 37.78 & 0.21 & 0.37 & 0.38 & 0.52 & 0.76 & 0.94 & 0.86 & 0.71 \\
\hline Schweden & 3.09 & 1685 & 0.87 & 38.00 & 0.16 & 0.33 & 0.36 & 0.59 & 0.80 & 0.97 & 0.90 & 0.71 \\
\hline Norwegen & 3.30 & 3889 & 0.90 & 34.62 & 0.13 & 0.37 & 0.36 & 0.48 & 0.80 & 0.98 & 0.86 & 0.70 \\
\hline Griechenland & 2.69 & 976 & 0.59 & 45.19 & 0.26 & 0.41 & 0.19 & 0.42 & 0.63 & 0.88 & 0.70 & 0.56 \\
\hline Spanien & 2.92 & 1053 & 0.66 & 40.49 & 0.21 & 0.33 & 0.23 & 0.34 & 0.59 & 0.89 & 0.77 & 0.44 \\
\hline Italien & 2.88 & 1164 & 0.79 & 38.64 & 0.14 & 0.33 & 0.24 & 0.44 & 0.71 & 0.95 & 0.67 & 0.53 \\
\hline Zypern & 3.23 & 1293 & 0.47 & 39.85 & 0.19 & 0.25 & 0.14 & 0.36 & 0.59 & 0.96 & 0.82 & 0.54 \\
\hline Malta & 3.06 & 916 & 0.51 & 40.75 & 0.20 & 0.32 & 0.21 & 0.35 & 0.84 & 0.99 & 0.88 & 0.57 \\
\hline Portugal & 3.02 & 655 & 0.76 & 41.84 & 0.21 & 0.26 & 0.17 & 0.32 & 0.62 & 0.94 & 0.73 & 0.50 \\
\hline Schweiz & 3.27 & 2876 & 0.87 & 37.81 & 0.15 & 0.31 & 0.28 & 0.61 & 0.77 & 0.98 & 0.83 & 0.69 \\
\hline
\end{tabular}




\begin{tabular}{|c|c|c|c|c|c|c|c|c|c|c|c|c|}
\hline Land & $\begin{array}{c}\text { Arbeits- } \\
\text { zufriedenheit } \\
\end{array}$ & Einkommen & Unbefristet & $\begin{array}{l}\text { Arbeits- } \\
\text { stunden } \\
\text { /Woche } \\
\end{array}$ & $\begin{array}{c}\text { Körperliche } \\
\text { Belastung } \\
\end{array}$ & $\begin{array}{c}\text { Ungünstige } \\
\text { Arbeitszeiten } \\
\end{array}$ & $\begin{array}{c}\text { Flexibilität } \\
\text { für } \\
\text { Arbeitgeber }\end{array}$ & $\begin{array}{c}\text { Flexibilität } \\
\text { für } \\
\text { Arbeitnehmer } \\
\end{array}$ & Autonomie & Sinnvoll & $\begin{array}{c}\text { Persönliche } \\
\text { Beziehungen } \\
\end{array}$ & $\begin{array}{c}\text { Anspruchs- } \\
\text { voll } \\
\end{array}$ \\
\hline Tschechien & 2.95 & 473 & 0.80 & 41.61 & 0.20 & 0.37 & 0.33 & 0.37 & 0.58 & 0.91 & 0.82 & 0.61 \\
\hline Estland & 2.82 & 348 & 0.86 & 40.33 & 0.22 & 0.36 & 0.34 & 0.40 & 0.72 & 0.91 & 0.86 & 0.65 \\
\hline Lettland & 2.76 & 260 & 0.86 & 41.59 & 0.21 & 0.36 & 0.29 & 0.39 & 0.73 & 0.94 & 0.87 & 0.60 \\
\hline Litauen & 2.77 & 266 & 0.84 & 40.60 & 0.24 & 0.32 & 0.22 & 0.28 & 0.69 & 0.88 & 0.79 & 0.50 \\
\hline Ungarn & 2.82 & 346 & 0.81 & 42.59 & 0.21 & 0.34 & 0.27 & 0.37 & 0.67 & 0.91 & 0.82 & 0.53 \\
\hline Polen & 2.90 & 338 & 0.70 & 44.18 & 0.21 & 0.39 & 0.29 & 0.41 & 0.65 & 0.95 & 0.76 & 0.55 \\
\hline Slowenien & 2.79 & 655 & 0.76 & 41.56 & 0.22 & 0.38 & 0.31 & 0.42 & 0.64 & 0.95 & 0.85 & 0.66 \\
\hline Slowakei & 2.88 & 309 & 0.84 & 43.13 & 0.21 & 0.40 & 0.32 & 0.31 & 0.60 & 0.93 & 0.85 & 0.59 \\
\hline Bulgarien & 2.73 & 132 & 0.73 & 44.40 & 0.20 & 0.37 & 0.20 & 0.25 & 0.57 & 0.95 & 0.79 & 0.59 \\
\hline Kroatien & 2.89 & 542 & 0.83 & 43.07 & 0.23 & 0.34 & 0.23 & 0.32 & 0.60 & 0.91 & 0.85 & 0.69 \\
\hline Rumänien & 2.65 & 142 & 0.87 & 46.24 & 0.24 & 0.42 & 0.29 & 0.45 & 0.63 & 0.94 & 0.76 & 0.59 \\
\hline Türkei & 2.47 & 316 & 0.22 & 54.35 & 0.23 & 0.48 & 0.20 & 0.54 & 0.80 & 0.86 & 0.56 & 0.48 \\
\hline
\end{tabular}

Hinweis: Detaillierte Informationen zur Definition der dargestellten Arbeitsmarktcharakteristika finden sich in Tabelle 9 im Anhang.

Quelle: Eigene Berechnungen basierend auf EWCS 2005; gewichtet. 
Tabelle 3: Korrelation auf Länderebene der durchschnittlichen Arbeitszufriedenheit mit durchschnittlichen Arbeitsmarktcharakteristika

\begin{tabular}{lclc}
\hline Merkmal & Korrelation & \multicolumn{1}{c}{ Merkmal } & Korrelation \\
\hline Einkommen & $\mathbf{0 . 7 7 2}$ & Flexibilität für Arbeitnehmer & $\mathbf{0 . 4 1 7}$ \\
& $(0.000)$ & & $(0.020)$ \\
unbefristeter Job & 0.273 & Autonomie & 0.262 \\
& $(0.138)$ & & $(0.154)$ \\
Arbeitsstunden / Woche & $\mathbf{- 0 . 8 0 9}$ & Sinnvolle Tätigkeit & $\mathbf{0 . 6 3 0}$ \\
& $(0.000)$ & & $(0.000)$ \\
Körperliche Belastung & $\mathbf{- 0 . 7 6 7}$ & Persönliche Beziehungen & $\mathbf{0 . 4 5 4}$ \\
& $(0.000)$ & & $(0.010)$ \\
Ungünstige Arbeitszeiten & $\mathbf{- 0 . 6 8 8}$ & Anspruchsvolle Tätigkeit & $\mathbf{0 . 4 7 2}$ \\
Flexibilität für Arbeitgeber & $(0.000)$ & & $(0.007)$ \\
& 0.242 & & $n=31$ \\
\hline
\end{tabular}

Hinweis: Detaillierte Informationen zur Definition der dargestellten Arbeitsmarktcharakteristika finden sich in Tabelle $9 \mathrm{im}$ Anhang. Korrelationen die auf einem $\alpha$-Fehlerniveau von 10\% signifikant sind, sind fett. Gewichtete Berechnung der Ländermittel(werte).

Quelle: Eigene Berechnungen basierend auf EWCS 2005; p-values in Klammern.

Diese bivariaten Ergebnisse auf nationaler Ebene können lediglich als erstes Indiz für mögliche Erklärungsmuster dienen. Um den Einfluss konkurrierender Faktoren zur Erklärung der Arbeitszufriedenheit zu isolieren und um Unterschiede in den Erklärungsmustern der Ländercluster zu identifizieren, wird im nachfolgenden Abschnitt eine multivariate Analyse der Arbeitszufriedenheit vorgenommen.

\section{Multivariate Analyse der Arbeitszufriedenheit}

Ziel der multivariaten empirischen Untersuchung ${ }^{9}$ ist es, zu bestimmen, welche Faktoren auf den Nutzen, den Personen aus der Arbeit ziehen, wirken und diese Einflussstärke zu quantifizieren. Dazu wird dem in der Literatur gängigen Modell (vgl. Clark und Oswald 1996) gefolgt, wonach sich dieser Nutzen als Funktion individueller Merkmale und Charakteristika der Arbeit ergibt. Der Nutzen $U_{\mathrm{i}}$ eines Arbeiters aus der Arbeit lässt sich demnach darstellen als:

$$
\mathrm{U}_{\mathrm{i}}=\mathrm{U}_{\mathrm{i}}\left(\mathrm{P}_{\mathrm{i}}, \mathrm{H}_{\mathrm{i}}, \mathrm{A}_{\mathrm{i}}, \mathrm{R}_{\mathrm{i}}\right)
$$

Dabei steht $\mathrm{P}$ für einen Vektor persönlicher Merkmale, H für einen Vektor von Merkmalen, die die Haushaltssituation des Individuums erfassen. A ist ein Vektor der Arbeitsplatzmerkmale und $\mathrm{R}$ beinhaltet Informationen über die Zugehörigkeit zu einem Land bzw. Ländercluster. Als Proxy für den Nutzen $U_{i}$, den eine Person aus der Arbeit zieht, dient die individuell 
angegebene Arbeitszufriedenheit gemessen auf einer Skala von 1 „gar nicht zufrieden“ bis 4 „sehr zufrieden“.

Für die vier diskreten Kategorien wird angenommen, dass sie die Realisation eines kontinuierlichen latenten Nutzenindex sind. Die beobachtete Arbeitszufriedenheit (JS ${ }_{\mathrm{i}}$ ) eines Individuums ist demnach wie folgt mit dem latenten Nutzenindex $\mathrm{JS}_{\mathrm{i}}^{*}$ verknüpft:

$$
\begin{aligned}
& \mathrm{JS}_{\mathrm{i}}=1 \quad \text { wenn } \quad \mathrm{JS}_{\mathrm{i}}^{*} \leq \mu_{1} \\
& \mathrm{JS}_{\mathrm{i}}=2 \quad \text { wenn } \mu_{1}<\mathrm{JS}_{\mathrm{i}}^{*} \leq \mu_{2} \\
& \mathrm{JS}_{\mathrm{i}}=3 \quad \text { wenn } \mu_{2}<\mathrm{JS}_{\mathrm{i}}^{*} \leq \mu_{3} \\
& \mathrm{JS}_{\mathrm{i}}=4 \quad \text { wenn } \mu_{3}<\mathrm{JS}_{\mathrm{i}}^{*}
\end{aligned}
$$

$\mu$ steht dabei für die Schwellenwerte, die zu einem nächsten Rangplatz führen.

Für den nicht beobachtbaren Nutzenindex $\mathrm{JS}_{\mathrm{i}}^{*}$ wird angenommen, dass er linear mit den Charakteristika eines Individuums verknüpft ist:

$$
\mathrm{JS}_{\mathrm{i}}^{*}=\mathrm{P}_{\mathrm{i}}^{\prime} \alpha+\mathrm{H}_{\mathrm{i}}^{\prime} \beta+\mathrm{A}_{\mathrm{i}}^{\prime} \gamma+\mathrm{R}_{\mathrm{i}}^{\prime} \delta+\varepsilon_{\mathrm{i}}
$$

$\alpha, \beta, \gamma$ und $\delta$ sind Koeffizientenvektoren. $\varepsilon$ ist ein standardnormalverteilter Störterm. Die Berücksichtigung des Wohlfahrtsregimes als erklärende Größe bedeutet, dass zunächst keine unterschiedlichen Erklärungsmuster in den Länderclustern zugelassen werden. Der Vektor R enthält Dummyvariablen für die in Abschnitt 4 definierten Ländercluster. Der Koeffizientenvektor $\delta$ kann also Niveauunterschiede in der Arbeitszufriedenheit nach Kontrolle für die anderen Erklärungsgrößen erfassen. Diese Unterschiede können sich durch die Ausgestaltung des Wohlfahrtsregimes ergeben oder auch kulturelle Niveauunterschiede im Verständnis der Zufriedenheitsskala sein.

Das hiermit beschriebene Modell wird als Ordered-Probit Regressionsmodell (McKelvey und Zavoina 1971, 1975) mit der Maximum Likelihood Methode geschätzt. Die verwendete Stichprobe umfasst abhängig Voll- und Teilzeitbeschäftigte mit einer Mindestwochenarbeitszeit von fünf Stunden im Alter von 15 bis 64 Jahren. Des Weiteren werden nur abhängig Beschäftigte betrachtet, da entscheidende Größen wie z.B. die arbeitgeberorientierte Flexibilität oder die Befristung des Beschäftigungsverhältnisses nur für diese Gruppe sinnvoll sind. Insgesamt wurden fünf Modelle mit unterschiedlichen Vektoren von Kontrollvariablen geschätzt (Tabelle 4). Eine Liste der im Sinne der Übersichtlichkeit hier nicht dargestellten Kontrollvariablen findet sich in Tabelle $10 \mathrm{im}$ Anhang. Ebenfalls hier nicht abgebildet sind die Schätzungen der drei Schwellenwerte (auf Nachfrage vom Autor erhältlich).

Die Effektstärke ist in nichtlinearen Modellen wie dem Ordered-Probit nicht direkt aus den Koeffizienten ablesbar, wohl aber ist die Wirkungsrichtung einer Erklärungsgröße zu erkennen. Ein positives Vorzeichen für einen Koeffizienten bedeutet, dass ein höherer Wert der erklärenden Größe ceteris paribus die Zufriedenheit erhöht. Ein negativer Koeffizient steht folglich für einen negativen Einfluss auf die Zufriedenheit. Eine Analyse der Effektstärken mittels marginaler Effekte geschieht im zweiten Teil dieses Abschnitts.

\section{Einkommen/Lohn}

Die objektive Einkommenssituation wird erfasst durch den logarithmierten Lohnsatz und eine Dummyvariable, die angibt, ob sich eine Person im ersten oder zweiten Einkommensdezil des Befragungslands befindet. Somit kann auch die relative Einkommensposition des Einzelnen 
berücksichtigt werden. Zudem wurde die subjektive Einschätzung, gut bezahlt zu werden, mit aufgenommen. Diese subjektive Einschätzung übt einen stark positiven und von der Modellspezifikation unabhängigen Einfluss auf die Arbeitszufriedenheit aus. Die Erklärung der Arbeitszufriedenheit mit einer anderen subjektiven Einschätzung ist jedoch problematisch. Werden beide Merkmale durch die Persönlichkeit des Individuums beeinflusst, so ist bei einer optimistischen Person zu erwarten, dass sie beide Merkmale positiv bewertet. Bei einer pessimistischen Person wären zwei negative Bewertungen zu erwarten. Die unterstellte Wirkungsrichtung, dass die Zufriedenheit mit der Bezahlung auf die Arbeitszufriedenheit wirkt, ist dann anzuzweifeln. Für eine Diskussion dieses Endogenitätsproblems siehe Hamermesh (2004).

Neben der subjektiven Einschätzung der Bezahlung wirkt auch der Lohnsatz signifikant positiv auf die Arbeitszufriedenheit. Für den Lohnsatz gilt zudem, dass dessen Einfluss deutlich ansteigt, wenn die subjektive Einschätzung nicht ins Modell aufgenommen wird (vgl. Spezifikation 3 und 4). Dass subjektive und objektive Erfassung des Lohns korreliert sind, trotzdem aber unterschiedliche Aspekte erfassen, wird durch das deutlich niedrigere Pseudo- $\mathrm{R}^{2}$ in Modell 4 ersichtlich. Die Zugehörigkeit zu den unteren beiden Dezilen hingegen übt keinen signifikanten negativen Einfluss aus.

\section{Arbeitszeiten}

Hinsichtlich der Arbeitszeiten zeigt sich, dass insbesondere ein Konflikt der Arbeitszeiten mit privaten Verpflichtungen stark negativ wirkt. Der Einfluss der Arbeitszeitflexibilität wird entscheidend durch deren Ausgestaltung beeinflusst. Flexible Arbeitszeitformen, die an den Interessen des Arbeitgebers orientiert sind (vom Arbeitgeber bestimmte Variation der Arbeitszeiten) wirken signifikant negativ auf die Arbeitszufriedenheit. Flexibilität der Arbeitszeiten, die hingegen vom Arbeitnehmer gestaltet werden kann, wirkt sich positiv auf die Arbeitszufriedenheit aus. Die starke Korrelation des Arbeitsumfangs (Anzahl Wochenstunden) mit der Arbeitszufriedenheit, die sich in Kapitel 5 auf Makroebene gezeigt hat, wird bei einer multivariaten Analyse auf Mikroebene nicht bestätigt.

\section{Körperliche Belastung und Art der Tätigkeit}

Ein besonders starker negativer Effekt zeigt sich, wenn Beschäftigte körperlichen Belastungen wie Lärm, Hitze, Kälte oder Vibrationen ausgesetzt sind. Dies bestätigt die bivariaten Ergebnisse auf Länderebene des vorangegangenen Abschnitts. Positiv auf die Arbeitszufriedenheit wirkt hingegen, wenn Arbeitnehmer ihre Aufgaben oder ihren Arbeitsablauf selbständig organisieren können, wenn Führungsverantwortung übernommen werden kann und besonders, wenn die ausgeübte Tätigkeit als sinnvoll erachtet wird.

\section{Arbeitsverhältnis und persönliche Beziehungen}

Wird die Einschätzung, ob der eigene Arbeitsplatz unsicher ist, berücksichtigt, so übt die Befristung von Arbeitsverhältnissen keinen negativen Einfluss auf die Arbeitszufriedenheit mehr aus. Gute Aufstiegsmöglichkeiten sowie gute Beziehungen zu Vorgesetzten und Kollegen hingegen erhöhen die Arbeitszufriedenheit. 
Tabelle 4: Ordered-Probit Regression der Arbeitszufriedenheit; Personen im Alter von 15-64 Jahren mit mind. 5 Arbeitsstunden/Woche

\begin{tabular}{|c|c|c|c|c|c|}
\hline & (1) & $\begin{array}{c}\text { (2) } \\
+ \text { Branchen } \\
+ \text { Tätigkeit }\end{array}$ & $\begin{array}{c}\text { (3) } \\
+ \text { Person } \\
+ \text { Haushalt } \\
\end{array}$ & $\begin{array}{c}\text { (4) } \\
\text { - subj. Eink } \\
\text { - 1./2. Dezil } \\
\end{array}$ & $\begin{array}{c}(5) \\
-1 . / 2 \text {. Dezil }\end{array}$ \\
\hline $\ln ($ Lohnsatz $)$ & $\begin{array}{r}0.087^{* * *} \\
(4.92)\end{array}$ & $\begin{array}{r}0.100^{* * *} \\
(5.54)\end{array}$ & $\begin{array}{r}0.118^{* * *} \\
(6.27)\end{array}$ & $\begin{array}{r}0.203^{* * *} \\
(11.47)\end{array}$ & $\begin{array}{r}0.120^{* * *} \\
(6.67)\end{array}$ \\
\hline Subjektiv gut bezahlt & $\begin{array}{l}0.550^{* * *} \\
(28.60)\end{array}$ & $\begin{array}{r}0.561^{* * *} \\
(28.98)\end{array}$ & $\begin{array}{r}0.558^{* * *} \\
(28.69)\end{array}$ & & $\begin{array}{r}0.558^{* * *} \\
(28.70)\end{array}$ \\
\hline 1. oder 2. Dezil & $\begin{array}{l}0.014 \\
\quad(0.53)\end{array}$ & $\begin{array}{l}0.006 \\
(0.22)\end{array}$ & $\begin{array}{r}-0.010 \\
(-0.37)\end{array}$ & & \\
\hline Stunden/Woche & $\begin{array}{r}-0.003 \\
(-1.06)\end{array}$ & $\begin{array}{l}-0.001 \\
(-0.24)\end{array}$ & $\begin{array}{l}0.002 \\
\quad(0.65)\end{array}$ & $\begin{array}{l}0.002 \\
(0.90)\end{array}$ & $\begin{array}{l}0.002 \\
\quad(0.82)\end{array}$ \\
\hline$(\mathrm{h} / \text { Woche })^{2} / 100$ & $\begin{array}{l}0.003 \\
\quad(1.04)\end{array}$ & $\begin{array}{l}0.002 \\
\quad(0.61)\end{array}$ & $\begin{array}{l}-0.000 \\
\quad(-0.07)\end{array}$ & $\begin{array}{l}0.001 \\
\quad(0.35)\end{array}$ & $\begin{array}{r}-0.001 \\
\quad(-0.15)\end{array}$ \\
\hline $\begin{array}{l}\text { Flexibilität für } \\
\text { Arbeitgeber }\end{array}$ & $\begin{array}{r}-0.124^{* *} \\
(-2.87)\end{array}$ & $\begin{array}{r}-0.140^{* *} \\
(-3.22)\end{array}$ & $\begin{array}{r}-0.114^{* *} \\
(-2.62)\end{array}$ & $\begin{array}{r}-0.128^{* *} \\
(-2.96)\end{array}$ & $\begin{array}{r}-0.115^{* *} \\
(-2.63)\end{array}$ \\
\hline $\begin{array}{l}\text { Flexibilität für } \\
\text { Arbeitnehmer }\end{array}$ & $\begin{array}{r}0.183^{* * *} \\
(6.48)\end{array}$ & $\begin{array}{r}0.213^{* * *} \\
(7.34)\end{array}$ & $\begin{array}{r}0.232^{* * *} \\
(7.92)\end{array}$ & $\begin{array}{r}0.266^{* * *} \\
(9.21)\end{array}$ & $\begin{array}{r}0.232^{* * *} \\
(7.92)\end{array}$ \\
\hline $\begin{array}{l}\text { Ungünstige Arbeits- } \\
\text { zeiten }\end{array}$ & $\begin{array}{r}-0.047 \\
(-1.51)\end{array}$ & $\begin{array}{r}-0.074^{*} \\
(-2.29)\end{array}$ & $\begin{array}{r}-0.083^{*} \\
(-2.55)\end{array}$ & $\begin{array}{r}-0.080^{*} \\
(-2.47)\end{array}$ & $\begin{array}{r}-0.083^{*} \\
(-2.55)\end{array}$ \\
\hline $\begin{array}{l}\text { Arbeitszeit/privat- } \\
\text { Konflikt }\end{array}$ & $\begin{array}{r}-0.496^{* * *} \\
(-21.15) \\
\end{array}$ & $\begin{array}{r}-0.495^{* * *} \\
(-21.05) \\
\end{array}$ & $\begin{array}{r}-0.471^{* * *} \\
(-19.84) \\
\end{array}$ & $\begin{array}{r}-0.507^{* * *} \\
(-21.56) \\
\end{array}$ & $\begin{array}{r}-0.471^{* * *} \\
(-19.85) \\
\end{array}$ \\
\hline Körperliche Belastung & $\begin{array}{r}-0.816^{* * *} \\
(-19.00)\end{array}$ & $\begin{array}{r}-0.794^{* * *} \\
(-16.25)\end{array}$ & $\begin{array}{r}-0.737^{* * *} \\
(-14.71)\end{array}$ & $\begin{array}{r}-0.796^{* * *} \\
(-15.97)\end{array}$ & $\begin{array}{r}-0.737^{* * *} \\
(-14.69)\end{array}$ \\
\hline Autonomie & $\begin{array}{r}0.113^{* * *} \\
(4.69)\end{array}$ & $\begin{array}{r}0.102^{* * *} \\
(4.14)\end{array}$ & $\begin{array}{r}0.110^{* * *} \\
(4.45)\end{array}$ & $\begin{array}{r}0.114^{* * *} \\
(4.63)\end{array}$ & $\begin{array}{r}0.110^{* * *} \\
(4.45)\end{array}$ \\
\hline Vorgesetzter & $\begin{array}{r}0.091^{* * *} \\
(3.58)\end{array}$ & $\begin{array}{r}0.110^{* * *} \\
(4.15)\end{array}$ & $\begin{array}{r}0.106^{* * *} \\
(3.94)\end{array}$ & $\begin{array}{r}0.127^{* * *} \\
(4.77)\end{array}$ & $\begin{array}{r}0.106^{* * *} \\
(3.94)\end{array}$ \\
\hline Anspruch & $\begin{array}{r}-0.064^{*} \\
(-2.24)\end{array}$ & $\begin{array}{r}-0.056^{+} \\
(-1.90)\end{array}$ & $\begin{array}{r}-0.017 \\
(-0.57)\end{array}$ & $\begin{array}{r}-0.033 \\
(-1.11)\end{array}$ & $\begin{array}{r}-0.017 \\
\quad(-0.56)\end{array}$ \\
\hline Sinn & $\begin{array}{r}0.813^{* * *} \\
(17.97) \\
\end{array}$ & $\begin{array}{r}0.797^{* * *} \\
(17.53) \\
\end{array}$ & $\begin{array}{r}0.785^{* * *} \\
(17.30) \\
\end{array}$ & $\begin{array}{r}0.826^{* * *} \\
(18.34) \\
\end{array}$ & $\begin{array}{r}0.785^{* * *} \\
(17.30) \\
\end{array}$ \\
\hline $\begin{array}{l}\text { Aufstiegsmöglich } \\
\text { keiten }\end{array}$ & $\begin{array}{r}0.383^{* * *} \\
(18.61)\end{array}$ & $\begin{array}{r}0.394^{* * *} \\
(18.94)\end{array}$ & $\begin{array}{r}0.397^{* * *} \\
(18.48)\end{array}$ & $\begin{array}{r}0.489^{* * *} \\
(23.14)\end{array}$ & $\begin{array}{r}0.398^{* * *} \\
(18.51)\end{array}$ \\
\hline Befristet beschäftigt & $\begin{array}{r}0.041+ \\
(1.81)\end{array}$ & $\begin{array}{r}0.041+ \\
(1.79)\end{array}$ & $\begin{array}{l}0.033 \\
\quad(1.41)\end{array}$ & $\begin{array}{l}0.038 \\
\quad(1.61)\end{array}$ & $\begin{array}{r}0.033 \\
\quad(1.39)\end{array}$ \\
\hline Arbeitsplatz unsicher & $\begin{array}{r}-0.304^{* * *} \\
(-12.61) \\
\end{array}$ & $\begin{array}{r}-0.297^{* * *} \\
(-12.26)\end{array}$ & $\begin{array}{r}-0.285^{* * *} \\
(-11.75) \\
\end{array}$ & $\begin{array}{r}-0.293^{* * *} \\
(-12.15)\end{array}$ & $\begin{array}{r}-0.286^{* * *} \\
(-11.77)\end{array}$ \\
\hline
\end{tabular}




\begin{tabular}{|c|c|c|c|c|c|}
\hline & (1) & $\begin{array}{c}\text { (2) } \\
+ \text { Branchen } \\
+ \text { Tätigkeit }\end{array}$ & $\begin{array}{c}\text { (3) } \\
+ \text { Person } \\
+ \text { Haushalt }\end{array}$ & $\begin{array}{c}\text { (4) } \\
\text { - subj. Eink } \\
-1 . / 2 . \text { Dezil }\end{array}$ & $\begin{array}{c}\text { (5) } \\
-1 . / 2 . \text { Dezil }\end{array}$ \\
\hline $\begin{array}{l}\text { Persönliche } \\
\text { Beziehungen }\end{array}$ & $\begin{array}{r}0.593^{* * *} \\
(15.86) \\
\end{array}$ & $\begin{array}{r}0.598^{* * *} \\
(15.93) \\
\end{array}$ & $\begin{array}{r}0.599^{* * *} \\
(15.88) \\
\end{array}$ & $\begin{array}{r}0.658^{* * *} \\
(17.58) \\
\end{array}$ & $\begin{array}{r}0.599^{* * *} \\
(15.88) \\
\end{array}$ \\
\hline \multicolumn{6}{|c|}{ Cluster (Ref. Skandinavien) } \\
\hline $\mathrm{UK}+$ Irland & $\begin{array}{r}-0.086^{*} \\
(-1.98)\end{array}$ & $\begin{array}{r}-0.076^{+} \\
(-1.74)\end{array}$ & $\begin{array}{r}-0.103^{*} \\
(-2.32)\end{array}$ & $\begin{array}{r}-0.052 \\
(-1.19)\end{array}$ & $\begin{array}{r}-0.103^{*} \\
(-2.34)\end{array}$ \\
\hline Kontinentaleuropa & $\begin{array}{r}-0.121^{* * *} \\
(-4.06)\end{array}$ & $\begin{array}{r}-0.121^{* * *} \\
(-4.03)\end{array}$ & $\begin{array}{c}-0.121^{* * *} \\
(-3.94)\end{array}$ & $\begin{array}{r}-0.029 \\
(-0.96)\end{array}$ & $\begin{array}{c}-0.120^{* * *} \\
(-3.92)\end{array}$ \\
\hline Südeuropa & $\begin{array}{r}-0.166^{* * *} \\
(-5.30)\end{array}$ & $\begin{array}{r}-0.146^{* * *} \\
(-4.60)\end{array}$ & $\begin{array}{r}-0.176^{* * *} \\
(-5.40)\end{array}$ & $\begin{array}{r}-0.093^{* *} \\
(-2.91)\end{array}$ & $\begin{array}{r}-0.175^{* * *} \\
(-5.39)\end{array}$ \\
\hline Osteuropa & $\begin{array}{r}-0.175^{* * *} \\
(-4.21)\end{array}$ & $\begin{array}{r}-0.141^{* * *} \\
(-3.32)\end{array}$ & $\begin{array}{r}-0.136^{* *} \\
(-3.10)\end{array}$ & $\begin{array}{l}-0.005 \\
\quad(-0.12)\end{array}$ & $\begin{array}{r}-0.133^{* *} \\
(-3.09)\end{array}$ \\
\hline Türkei & $\begin{array}{r}-0.246^{* *} \\
(-3.15) \\
\end{array}$ & $\begin{array}{r}-0.229^{* *} \\
(-2.92) \\
\end{array}$ & $\begin{array}{r}-0.221^{* *} \\
(-2.75) \\
\end{array}$ & $\begin{array}{r}-0.077 \\
(-0.96) \\
\end{array}$ & $\begin{array}{r}-0.217^{* *} \\
(-2.73) \\
\end{array}$ \\
\hline Branche & & $\checkmark$ & $\checkmark$ & $\checkmark$ & $\checkmark$ \\
\hline Tätigkeit & & $\checkmark$ & $\checkmark$ & $\checkmark$ & $\checkmark$ \\
\hline Person und Haushalt & & & $\checkmark$ & $\checkmark$ & $\checkmark$ \\
\hline Pseudo $R^{2}$ & 0.159 & 0.162 & 0.168 & 0.148 & 0.168 \\
\hline $\mathrm{N}$ & 18766 & 18766 & 18766 & 18766 & 18766 \\
\hline
\end{tabular}

Hinweis: Detaillierte Informationen zur Definition der dargestellten Arbeitsmarktcharakteristika finden sich in Tabelle 9 im Anhang. Robuste Standardfehler, t-Statistiken in Klammern. ${ }^{+} p<0.10,{ }^{*} p<0.05,{ }^{* *} p<0.01,{ }^{* * *} p$ $<0.001$.

Quelle: Eigene Berechnungen basierend auf EWCS 2005.

\section{Wohlfahrtsregime}

Wird für die bereits diskutierten Faktoren kontrolliert, so ergibt sich für die skandinavischen Staaten das höchste Niveau an Arbeitszufriedenheit. Alle anderen Ländercluster weisen im Vergleich dazu eine signifikant niedrigere Arbeitszufriedenheit auf. Während Großbritannien, Irland und die kontinentaleuropäischen Staaten einen vergleichbaren Abstand aufweisen, fällt der Abstand zur Türkei deutlich größer aus. Dies deutet darauf hin, dass entscheidende nicht beobachtete Faktoren existieren, die in Bezug zu den betrachteten Länderclustern stehen.

\section{Multivariate Analyse getrennt nach Wohlfahrtsregimen}

Um nun genauer zu analysieren, ob sich die Erklärungsmuster der Arbeitszufriedenheit in den unterschiedlichen Wohlfahrtsregimen mit zum Teil auch deutlich variierenden Wohlstandsniveaus und Arbeitsbedingungen unterscheiden, wird der oben verwendete Ansatz erweitert:

$\mathrm{JS}_{\mathrm{i}}^{*}=\mathrm{P}_{\mathrm{i}}^{\prime} \alpha_{\mathrm{k}}+\mathrm{H}_{\mathrm{i}}^{\prime} \beta_{\mathrm{k}}+\mathrm{A}_{\mathrm{i}}^{\prime} \gamma_{\mathrm{k}}+\mathrm{R}_{\mathrm{i}}^{\prime} \delta_{\mathrm{k}}+\varepsilon_{\mathrm{i}}$

Dabei steht der Index k der Koeffizientenvektoren für die Wohlfahrtsregimes. Für jedes Regime wird ein eigener Koeffizientenvektor geschätzt. Unterscheiden sich die Erklärungsmuster nicht, so müsste der Einfluss der Regressanden für alle k ähnlich ausgeprägt sein. Da in der Türkei nicht ausreichend abhängig Beschäftigte für eine eigene Schätzung vorliegen, wird 
abweichend von der bisherigen Ländergruppierung nun eine gemeinsame Schätzung für die Türkei und die osteuropäischen Staaten durchgeführt. Im Weiteren wird diese Abgrenzung als Bonoli +1 bezeichnet.

Ein direkter Vergleich der Koeffizienten der fünf in Tabelle 5 dargestellten Schätzungen ist in einem nicht-linearen Modell wie dem Ordered-Probit nicht zulässig. Die geschätzten Koeffizienten beschreiben Veränderungen der latenten Variable $\mathrm{JS}_{\mathrm{i}}^{*}$ bei einer Änderung der erklärenden Größen. Die Identifikation der Koeffizienten bei einer Ordered-Probit Schätzung erfolgt durch die Festlegung der Störtermverteilung (Standardnormalverteilung). ${ }^{10}$ Lage und Varianz des latenten Zufriedenheitsindex können nicht beobachtet werden. Es ergibt sich jedoch aus den zur Schätzung getroffenen Annahmen eine Verteilung der geschätzten latenten Variable $\mathrm{JS}_{\mathrm{i}}^{*}, \widehat{\mathrm{JS}}_{\mathrm{i}}^{*}$. Eine Änderung der Modellspezifikation oder unterschiedliche zur Schätzung verwendete Stichproben führen zu unterschiedlichen Verteilungen für $\widehat{\mathrm{JS}}_{\mathrm{i}}^{*}$ In der Folge variiert auch die Größe der geschätzten Koeffizienten über verschiedene Modellspezifikationen oder Stichproben (Long 1997: 70).

Für den hier vorgenommenen Vergleich von Regressionsergebnissen über mehrere Gruppen, deren Arbeitszufriedenheit in unterschiedlichem Ausmaß durch das Modell erklärt werden kann, bedeutet dies, dass die Koeffizienten Änderungen in unterschiedlich skalierten Zufriedenheitsindizes beschreiben. Für die Schätzungen in Tabelle 5, die auf sehr heterogenen Ländergruppen basieren, ist ein direkter Vergleich der Koeffizienten also nicht möglich. Die Signifikanz und die Wirkungsrichtung, erkennbar am Vorzeichen der Koeffizienten, sind jedoch interpretierbar.

An den Ergebnissen lässt sich erkennen, dass clusterübergreifend ein signifikant positiver Effekt der subjektiven Wahrnehmung gut bezahlt zu sein, der arbeitnehmerorientierten Arbeitszeitflexibilität, der persönlichen Beziehungen, der Aufstiegsmöglichkeiten und für eine als sinnvoll wahrgenommene Tätigkeit existiert. Über alle Ländergruppen hinweg negativ wirkt die körperliche Belastung am Arbeitsplatz, die subjektive Einschätzung, dass der Arbeitsplatz gefährdet ist und Konflikte zwischen beruflichen und privaten Verpflichtungen.

Unterschiede für die Ländergruppen zeigen sich insbesondere für die Autonomie am Arbeitsplatz. Hier besteht nur in Staaten mit einem relativ hohen Einkommensniveau (Großbritannien, Irland, Skandinavien und Kontinentaleuropa) ein positiver Effekt auf die Arbeitszufriedenheit. Für Süd- und Osteuropa wie die Türkei zeigt sich dieser Effekt nicht. Dies ist ein Indiz dafür, dass bestimmte ,weiche“ Faktoren der Arbeitsbedingungen erst dann eine Rolle spielen, wenn ein gewisses Wohlstandsniveau erreicht ist. Dies bestätigt das Ergebnis von Origo \& Pagani (2006), die einen signifikanten Einfluss der Autonomie nur für Nordeuropäische Staaten gefunden haben. Ähnliches gilt für die Führungsverantwortung, die nur in Osteuropa und der Türkei keinen positiven Einfluss auf die Zufriedenheit hat.

10 Durch die Festlegung der Störtermverteilung wird die Varianz der latenten Variable bestimmt. Zur Identifikation ist auch noch eine Annahme zur Lage der Verteilung zu treffen. In Stata geschieht dies durch die Schätzung ohne Konstante. Andere Programmpakete schätzen mit Konstante und ermöglichen die Identifikation des Modells, indem $\mu 1$ auf den Wert 0 gesetzt wird. 
Tabelle 5: Ordered-Probit Regression der Arbeitszufriedenheit für Bonoli+1 Ländergruppen, Alter 15-64, Voll- und Teilzeit mit mindestens 5 h

\begin{tabular}{|c|c|c|c|c|c|}
\hline & $\begin{array}{c}\text { (1) } \\
\text { UK und } \\
\text { Irland }\end{array}$ & $\begin{array}{c}\text { (2) } \\
\text { Kontinental- } \\
\text { europa }\end{array}$ & $\begin{array}{c}\text { (3) } \\
\text { Skandi- } \\
\text { navien }\end{array}$ & $\begin{array}{c}\text { (4) } \\
\text { Südeuropa }\end{array}$ & $\begin{array}{c}\text { (5) } \\
\text { Osteuropa } \\
+ \text { Türkei }\end{array}$ \\
\hline $\ln ($ Lohnsatz $)$ & $\begin{array}{l}-0.060 \\
\quad(-0.38)\end{array}$ & $\begin{array}{r}-0.351^{* * *} \\
(-4.65)\end{array}$ & $\begin{array}{r}0.271^{* * *} \\
\quad(5.15)\end{array}$ & $\begin{array}{l}0.061 \\
\quad(1.37)\end{array}$ & $\begin{array}{r}0.149^{* * *} \\
\quad(5.34)\end{array}$ \\
\hline Subjektiv gut bezahlt & $\begin{array}{r}0.654^{* * *} \\
(8.47)\end{array}$ & $\begin{array}{r}0.526^{* * *} \\
(12.26)\end{array}$ & $\begin{array}{r}0.412^{* * *} \\
(9.02)\end{array}$ & $\begin{array}{r}0.630^{* * *} \\
(15.00)\end{array}$ & $\begin{array}{l}0.645^{* * *} \\
\quad(18.39)\end{array}$ \\
\hline 1. oder 2. Dezil & $\begin{array}{l}0.050 \\
\quad(0.36) \\
\end{array}$ & $\begin{array}{r}-0.119 \\
(-1.53) \\
\end{array}$ & $\begin{array}{l}0.140^{*} \\
(2.04) \\
\end{array}$ & $\begin{array}{l}0.096 \\
\quad(1.54) \\
\end{array}$ & $\begin{array}{r}-0.115^{* *} \\
(-2.73) \\
\end{array}$ \\
\hline Stunden/Woche & $\begin{array}{l}-0.021 \\
\quad(-1.15)\end{array}$ & $\begin{array}{l}-0.018 \\
\quad(-1.49)\end{array}$ & $\begin{array}{l}0.001 \\
\quad(0.15)\end{array}$ & $\begin{array}{l}0.009 \\
\quad(1.18)\end{array}$ & $\begin{array}{l}0.001 \\
\quad(0.29)\end{array}$ \\
\hline$(\mathrm{h} / \text { Woche })^{2} / 100$ & $\begin{array}{l}0.026 \\
\quad(1.17)\end{array}$ & $\begin{array}{l}0.013 \\
\quad(0.77)\end{array}$ & $\begin{array}{l}0.006 \\
\quad(0.49)\end{array}$ & $\begin{array}{r}-0.006 \\
\quad(-0.61)\end{array}$ & $\begin{array}{r}-0.000 \\
\quad(-0.04)\end{array}$ \\
\hline Flexibilität für Arbeitgeber & $\begin{array}{l}0.101 \\
\quad(0.56)\end{array}$ & $\begin{array}{l}-0.210^{*} \\
(-2.08)\end{array}$ & $\begin{array}{l}0.005 \\
\quad(0.05)\end{array}$ & $\begin{array}{l}-0.051 \\
\quad(-0.47)\end{array}$ & $\begin{array}{r}-0.160^{*} \\
(-2.26)\end{array}$ \\
\hline Flexibilität für Arbeitnehmer & $\begin{array}{r}0.340^{* *} \\
(2.91)\end{array}$ & $\begin{array}{r}0.186^{* *} \\
(2.84)\end{array}$ & $\begin{array}{r}0.251^{* * *} \\
(3.61)\end{array}$ & $\begin{array}{r}0.281^{* * *} \\
(4.17)\end{array}$ & $\begin{array}{r}0.289^{* * *} \\
(5.45)\end{array}$ \\
\hline Ungünstige Arbeitszeiten & $\begin{array}{r}-0.034 \\
(-0.25)\end{array}$ & $\begin{array}{r}-0.074 \\
(-0.96)\end{array}$ & $\begin{array}{r}-0.119 \\
(-1.56)\end{array}$ & $\begin{array}{r}-0.107 \\
(-1.37)\end{array}$ & $\begin{array}{l}-0.050 \\
\quad(-0.95)\end{array}$ \\
\hline Arbeitszeit/privat-Konflikt & $\begin{array}{r}-0.510^{* * *} \\
(-5.22) \\
\end{array}$ & $\begin{array}{r}-0.576^{* * *} \\
(-9.74) \\
\end{array}$ & $\begin{array}{r}-0.500^{* * *} \\
(-7.53) \\
\end{array}$ & $\begin{array}{r}-0.377^{* * *} \\
(-7.48) \\
\end{array}$ & $\begin{array}{c}-0.474^{* * *} \\
(-12.82) \\
\end{array}$ \\
\hline Körperliche Belastung & $\begin{array}{r}-0.508^{*} \\
(-2.10)\end{array}$ & $\begin{array}{r}-0.708^{* * *} \\
(-5.95)\end{array}$ & $\begin{array}{r}-0.728^{* * *} \\
(-5.34)\end{array}$ & $\begin{array}{r}-0.823^{* * *} \\
(-7.57)\end{array}$ & $\begin{array}{r}-0.761^{* * *} \\
(-9.65)\end{array}$ \\
\hline Autonomie & $\begin{array}{r}0.311^{* *} \\
(3.06)\end{array}$ & $\begin{array}{r}0.173^{* *} \\
(3.05)\end{array}$ & $\begin{array}{r}0.269^{* * *} \\
(3.61)\end{array}$ & $\begin{array}{l}0.026 \\
\quad(0.47)\end{array}$ & $\begin{array}{l}0.055 \\
\quad(1.38)\end{array}$ \\
\hline Vorgesetzter & $\begin{array}{r}0.214^{*} \\
(1.99)\end{array}$ & $\begin{array}{r}0.133^{*} \\
(2.28)\end{array}$ & $\begin{array}{r}0.153^{*} \\
(2.21)\end{array}$ & $\begin{array}{r}0.143^{*} \\
(2.47)\end{array}$ & $\begin{array}{r}-0.000 \\
\quad(-0.01)\end{array}$ \\
\hline Anspruch & $\begin{array}{r}-0.006 \\
(-0.04)\end{array}$ & $\begin{array}{l}-0.101 \\
\quad(-1.45)\end{array}$ & $\begin{array}{l}0.062 \\
\quad(0.76)\end{array}$ & $\begin{array}{l}0.055 \\
\quad(0.83)\end{array}$ & $\begin{array}{r}-0.066 \\
\quad(-1.40)\end{array}$ \\
\hline Sinn & $\begin{array}{r}0.489^{* *} \\
(2.64) \\
\end{array}$ & $\begin{array}{r}0.737^{* * *} \\
(6.27) \\
\end{array}$ & $\begin{array}{r}1.300^{* * *} \\
(9.01) \\
\end{array}$ & $\begin{array}{r}0.780^{* * *} \\
(7.63) \\
\end{array}$ & $\begin{array}{r}0.772^{* * *} \\
(11.94) \\
\end{array}$ \\
\hline Aufstiegsmöglichkeiten & $\begin{array}{r}0.356^{* * *} \\
(4.43)\end{array}$ & $\begin{array}{r}0.399^{* * *} \\
(8.21)\end{array}$ & $\begin{array}{r}0.308^{* * *} \\
(5.99)\end{array}$ & $\begin{array}{r}0.434^{* * *} \\
(9.38)\end{array}$ & $\begin{array}{r}0.421^{* * *} \\
(10.91)\end{array}$ \\
\hline Befristet beschäftigt & $\begin{array}{r}-0.079 \\
\quad(-0.95)\end{array}$ & $\begin{array}{l}0.067 \\
\quad(1.04)\end{array}$ & $\begin{array}{l}0.036 \\
\quad(0.55)\end{array}$ & $\begin{array}{r}0.094^{*} \\
(1.97)\end{array}$ & $\begin{array}{r}-0.009 \\
\quad(-0.22)\end{array}$ \\
\hline Arbeitsplatz unsicher & $\begin{array}{r}-0.513^{* * *} \\
(-4.07)\end{array}$ & $\begin{array}{r}-0.301^{* * *} \\
(-4.60) \\
\end{array}$ & $\begin{array}{r}-0.238^{* * *} \\
(-3.47)\end{array}$ & $\begin{array}{r}-0.292^{* * *} \\
(-5.40) \\
\end{array}$ & $\begin{array}{r}-0.262^{* * *} \\
(-7.35) \\
\end{array}$ \\
\hline
\end{tabular}




\begin{tabular}{lccccc}
\hline & $\begin{array}{c}(\mathbf{1}) \\
\text { UK und } \\
\text { Irland }\end{array}$ & $\begin{array}{c}\text { Kontinental- } \\
\text { europa }\end{array}$ & $\begin{array}{c}\text { (3) } \\
\text { Skandi- } \\
\text { navien }\end{array}$ & $\begin{array}{c}\text { Südeuropa } \\
\text { (5) }\end{array}$ & $\begin{array}{c}\text { Osteuropa } \\
\text { + Türkei }\end{array}$ \\
\hline Persönliche Beziehungen & $0.605^{* *}$ & $0.552^{* * *}$ & $0.709^{* * *}$ & $0.608^{* * *}$ & $0.614^{* * *}$ \\
& $(2.87)$ & $(7.06)$ & $(5.94)$ & $(7.85)$ & $(9.84)$ \\
\hline Branche & $\checkmark$ & $\checkmark$ & $\checkmark$ & $\checkmark$ & $\checkmark$ \\
Tätigkeit & $\checkmark$ & $\checkmark$ & $\checkmark$ & $\checkmark$ & $\checkmark$ \\
Person und Haushalt & $\checkmark$ & $\checkmark$ & $\checkmark$ & 0.174 & 0.177 \\
\hline Pseudo $R^{2}$ & 0.169 & 0.147 & 0.137 & 3772 & 6957 \\
$N$
\end{tabular}

Hinweis: Detaillierte Informationen zur Definition der dargestellten Arbeitsmarktcharakteristika finden sich in Tabelle 9 im Anhang. Robuste Standardfehler, t-Statistiken in Klammern. ${ }^{+} p<0.10,{ }^{*} p<0.05,{ }^{* *} p<0.01,{ }^{* * *} p$ $<0.001$.

Quelle: Eigene Berechnungen basierend auf EWCS 2005.

\section{Zu den Effektstärken einzelner Erklärungsfaktoren}

Die Interpretation der Ergebnisse bis hierher hat nur auf die Wirkungsrichtung und Signifikanz der Einflüsse Bezug genommen. Um darüber hinaus die Effektstärken einzelner Variablen vergleichen zu können, werden nun die marginalen Effekte der Variablen bestimmt und gegenübergestellt. Marginale Effekte werden dabei für alle dargestellten Merkmale bestimmt, die das Einkommen, die Tätigkeit und die Arbeitsbedingungen der Probanden erfassen. Betrachtet wird dabei, wie sich die Wahrscheinlichkeit einer Person „sehr zufrieden“ zu sein ändert, wenn ceteris paribus eine erklärende Variable verändert wird.

Die vorgenommenen Veränderungen der erklärenden Größen werden in Tabelle 6 zusammengefasst. Für den Lohnsatz wurde der Effekt bestimmt, der aus einem Anstieg des Einkommens vom ersten auf den neunten Dezilwert resultiert. Für die Wochenarbeitszeit wurde ein Anstieg von 35 auf 45 Wochenstunden betrachtet. Bezüglich der körperlichen Belastung wurde ein Wert von 0 (keinen körperlichen Belastungen ausgesetzt) mit einem Wert von 0,5 (fünf von zehn körperlichen Belastungen ausgesetzt) verglichen, da es nicht realistisch ist, allen zehn erfassten Belastungen ausgesetzt zu sein. Bei allen verbleibenden Merkmalen wurde ein Kontrast der Ausprägungen 0 und 1 berechnet.

Da sich diese Effekte von Person zu Person unterscheiden können, wird in jeder Länderklasse eine „Durchschnittsperson“ kreiert, bei der alle Merkmale jeweils den Durchschnittswert des jeweiligen Clusters annehmen.

Tabelle 6: Kontraste zur Bestimmung der marginalen Effekte auf $\mathrm{P}(\mathrm{Y}=4)$

\begin{tabular}{llll}
\hline Merkmal & Kontrast & Merkmal & Kontrast \\
\hline ln(Lohnsatz) & 1. gegen 9. Dezil & Autonomie & 0 gegen 1 \\
Subjektiv gut bezahlt ${ }^{1}$ & 0 gegen 1 & Vorgesetzter & 0 gegen 1 \\
1. oder 2. Dezil & 0 gegen 1 & Anspruch & 0 gegen 1 \\
Arbeitsstunden/Woche & 35 gegen 45 & Sinn & 0 gegen 1 \\
Flexibilität für Arbeitgeber & 0 gegen 1 & Aufstiegsmöglichkeiten & 0 gegen 1 \\
Flexibilität für Arbeitnehmer & 0 gegen 1 & Befristete Beschäftigung & 0 gegen 1
\end{tabular}




\begin{tabular}{llll}
\hline Merkmal & Kontrast & Merkmal & Kontrast \\
\hline Ungünstige Arbeitszeiten & 0 gegen 1 & Arbeitsplatz unsicher & 0 gegen 1 \\
Arbeitszeit/privat-Konflikt & 0 gegen 1 & Persönliche Beziehungen & 0 gegen 1 \\
Körperliche Belastung & 0 gegen 0,5 & & \\
\hline
\end{tabular}

Hinweis: Detaillierte Informationen zur Definition der dargestellten Arbeitsmarktcharakteristika finden sich in Tabelle 9 im Anhang.

Quelle: Eigene Zusammenstellung..

Eine Rangliste der zehn Arbeitsplatzmerkmale, die die stärksten Veränderungen der Wahrscheinlichkeit „sehr zufrieden“ mit der Arbeit zu sein verursachen, zeigt Tabelle 7. Die Absolutbeträge der Veränderungen für die zehn bedeutendsten Einflussgrößen sind in Tabelle 11 im Anhang zusammengefasst. Die deutlich ersichtlichen Unterschiede der Veränderungen in den Ländergruppen sind durch das unterschiedliche Niveau der Arbeitszufriedenheit zu erklären. Würde statt der Veränderung von $\mathrm{P}(\mathrm{Y}=4)$ die Veränderung von $\mathrm{P}(\mathrm{Y}=1)$ betrachtet werden, so ergäben sich die größten Wahrscheinlichkeitsveränderungen für Staaten mit niedriger Arbeitszufriedenheit. Ein direkter Vergleich der absoluten Beträge der Veränderungen (von $\mathrm{P}(\mathrm{Y}=4)$ ) über mehrere Cluster wäre irreführend, da nur ein Ausschnitt der Zufriedenheitsskala betrachtet wird. Im Folgenden wird deshalb auf einen aussagekräftigeren Vergleich der Rangfolgen der Effektstärken Bezug genommen.

Tabelle 7: Ranglisten marginaler Effekte auf $\mathrm{P}(\mathrm{Y}=4)$ für Bonoli+1 Cluster

\begin{tabular}{rlllll}
\hline Rang & UK und Irland & Kontinental & Skandinavien & Südeuropa & Osteuropa + Türkei \\
\hline $\mathbf{1}$ & subj. gut bezahlt & Sinn & Sinn & subj. gut bezahlt & subj. gut bezahlt \\
$\mathbf{2}$ & AZ/privat-Konflikt & subj. gut bezahlt & AZ/privat-Konflikt & Sinn & Sinn \\
$\mathbf{3}$ & Arbeitspl. unsicher & AZ/privat-Konflikt & subj. gut bezahlt & Aufstiegsmögl. & Aufstiegsmögl. \\
$\mathbf{4}$ & Sinn & Aufstiegsmögl. & In(Lohnsatz) & AZ/privat-Konflikt & AZ/privat-Konflikt \\
$\mathbf{5}$ & Aufstiegsmögl. & körperl. Belastung & körperl. Belastung & körperl. Belastung & körperl. Belastung \\
$\mathbf{6}$ & Flex AN & Flex AN & Aufstiegsmögl. & Flex AN & Flex AN \\
$\mathbf{7}$ & pers. Bezieh. & Arbeitspl. unsicher & Flex AN & Arbeitspl. unsicher & In(Lohnsatz) \\
$\mathbf{8}$ & Autonomie & In(Lohnsatz) & pers. Bezieh. & pers. Bezieh. & pers. Bezieh. \\
$\mathbf{9}$ & körperl. Belastung & pers. Bezieh. & Autonomie & Vorgesetzter & Arbeitspl. unsicher \\
$\mathbf{1 0}$ & Vorgesetzter & Autonomie & Arbeitspl. unsicher & befristet & Flex. AG \\
\hline
\end{tabular}

Hinweis: Detaillierte Informationen zur Definition der dargestellten Arbeitsmarktcharakteristika finden sich in Tabelle 9 im Anhang. Die Berechnung der marginalen Effekte, die dieser Tabelle zugrunde liegen, erfolgte für eine hypothetische Person, die bei allen Merkmalen die Durchschnittswerte des jeweiligen Clusters aufweist. Quelle: Eigene Berechnungen basierend auf EWCS 2005.

Die finanziellen Aspekte betreffend fällt auf, dass in allen Gruppen die subjektive Einschätzung, gut bezahlt zu sein, unter den drei stärksten Einflüssen ist. Nur für Skandinavien und Kontinentaleuropa, also Staaten mit ausgeprägter sozialer Absicherung und einem hohen Wohlstandsniveau, ist dies nicht der stärkste Einfluss. Für diese Gruppe gilt, dass die Einschätzung, einer sinnvollen Tätigkeit nachzugehen, den stärksten Einfluss ausübt. Dieses Ergebnis ist einerseits in Übereinstimmung mit den Ergebnissen von Sousa-Poza und SousaPoza (2000) im Hinblick auf die große Bedeutung des Einkommens (subjektiv) in Osteuropa, widerspricht diesen aber auch im Hinblick auf Großbritannien. Für Großbritannien finden Sousa-Poza und Sousa-Poza (2000) keinen signifikanten Einkommenseffekt. Die Ergebnisse dieser Arbeit weisen jedoch für Großbritannien und Irland auch nach Kontrolle für den Lohn- 
satz die subjektive Einschätzung, gut bezahlt zu sein, als stärksten Einflussfaktor aus. Die in unserer Studie gefundene clusterübergreifend bedeutende Rolle des Einkommens widerspricht auch der Einschätzung von Clark (2005), dass das Interesse an der ausgeübten Tätigkeit und die Arbeitsplatzsicherheit die entscheidenden Faktoren sind und das Einkommen und die Arbeitszeiten dabei eine untergeordnete Rolle spielen.

Bezüglich der Arbeitszeiten zeigt sich ein ebenfalls starker Effekt, wenn die Arbeitszeit mit privaten Verpflichtungen kollidiert. Dabei fällt auf, dass dieser Effekt in Südeuropa, Osteuropa und der Türkei weniger stark ausfällt als in den übrigen Gruppen. Dies entspricht dem Ergebnis von Origo und Pagani (2006), dass in Kontinentaleuropa im Vergleich mit Südeuropa flexible Arbeitszeiten besonders negativ auf die Arbeitszufriedenheit wirken.

Der von Sousa-Poza und Sousa-Poza (2000) gefundene variierende Effekt der Arbeitsplatzsicherheit wird auch hier bestätigt. In Großbritannien und Irland spielt die Sicherheit des Arbeitsplatzes eine größere Rolle als im Rest der betrachteten Länder. Die Autonomie bei der Organisation der Arbeitsaufgaben findet sich nur in Staaten mit hohem Wohlstandsniveau (Großbritannien, Irland, Kontinentaleuropa und Skandinavien) unter den zehn stärksten Einflüssen; ebenfalls in Übereinstimmung mit Sousa-Poza und Sousa-Poza (2000).

Unsere Studie mit Verwendung einer Datenbasis, die auf europäischer Ebene einheitliche und detaillierte Informationen über die Charakteristika der Arbeitsstelle und sozioökonomische Merkmale (insbesondere auch objektive und subjektive Einkommensinformationen) umfasst, bestätigt Ergebnisse der bereits vorhandenen Literatur, widerspricht diesen aber auch in Teilbereichen. Insgesamt stützen die unterschiedlichen Ergebnisse für Staaten mit hohem und niedrigerem Wohlstandsniveau die These, dass die Arbeitszufriedenheit nicht durch ein einheitliches Erklärungsmuster abgebildet werden kann. Ferner deuten die Unterschiede zwischen Großbritannien, Irland und Südeuropa einerseits und Skandinavien und Kontinentaleuropa andererseits darauf hin, dass auch das Ausmaß der sozialen Absicherung zu divergierenden Erklärungsmustern führt.

\section{Zusammenfassung}

Ziel dieser Analyse ist der europäische Vergleich des Arbeitszufriedenheitsniveaus und die Überprüfung, ob unterschiedliche Wohlfahrtsregimes, Wohlfahrtsniveaus und Arbeitsbedingungen zu Unterschieden in den Bestimmungsfaktoren der Arbeitszufriedenheit führen. $\mathrm{Zu}$ diesem Zweck wird auf Daten aus dem European Working Conditions Survey 2005 mit einem einheitlichen Design für 31 europäische Länder zurückgegriffen. Erstmals kann damit ein umfassender europäischer Vergleich durchgeführt werden, der auch Osteuropa und die Türkei mit berücksichtigt. Der besondere Beitrag dieser Studie zur existierenden Literatur ist die Verwendung dieser international vergleichbaren Datenbasis, die sowohl über detaillierte Informationen zu den Arbeitsbedingungen als auch über die Haushaltssituation, persönliche Merkmale und über objektive sowie subjektive Einkommensinformationen mit gleichen Items für die europäischen Länder verfügt. Um eine übersichtliche Darstellung der Ergebnisse zu ermöglichen, wurden Ländergruppen gebildet, die in der Ausgestaltung der Wohlfahrtsregimes, im Wohlfahrtsniveau und in den Arbeitsbedingungen weitgehend homogen sind. Diese auf Esping-Andersen (1990) und Bonoli (1997) basierende Klassifikation der Länder bringt als Ergebnis insbesondere in der multivariaten Analyse Unterschiede und Gemeinsamkeiten in den Erklärungsmustern der Arbeitszufriedenheit über Ländercluster hinweg.

Die deskriptive Analyse zeigt, dass das höchste Arbeitszufriedenheitsniveau in Großbritannien, Irland und den skandinavischen Staaten erreicht wird. Am Ende der Rangliste finden 
sich überwiegend osteuropäische Staaten, Griechenland und die Türkei. Unser internationaler Vergleich der Arbeitsbedingungen liefert erste Erkenntnisse über entscheidende Determinanten der Arbeitszufriedenheit. Die Staaten an der Spitze zeichnen sich durch ein hohes Einkommensniveau und moderate Arbeitszeiten aus, wohingegen in Süd- und Osteuropa und der Türkei deutlich geringere Einkommen bei höherer Wochenarbeitszeit erzielt werden. Auch die körperlichen Belastungen durch die Arbeit fallen dort stärker aus. Bei der Korrelation der durchschnittlichen Arbeitsbedingungen auf Länderebene mit der Arbeitszufriedenheit zeigen sich die betragsmäßig stärksten Korrelationen der Arbeitszufriedenheit mit der körperlichen Belastung durch die Arbeit und die Wochenarbeitsstunden. Die Meinung einer sinnvollen Tätigkeit nachzugehen oder die persönlichen Beziehungen am Arbeitsplatz korrelieren ebenfalls mit der Arbeitszufriedenheit, jedoch weniger ausgeprägt. Dieses Ergebnis steht in Widerspruch zu vielen Querschnittsanalysen auf Mikroebene (z.B. Sousa-Poza und Sousa-Poza 2000, Clark 2005).

Um konkurrierende Bestimmungsfaktoren der Arbeitszufriedenheit auf individueller Ebene zu identifizieren wird in dieser Studie ein Ordered-Probit Regressionsmodell geschätzt. In einem ersten Schritt werden dabei Regressionsmodelle für alle Staaten gemeinsam spezifiziert. Als starke Einflüsse auf die Arbeitszufriedenheit werden dabei der Lohnsatz, die subjektive Einschätzung gut bezahlt zu sein, die Ausgestaltung der Arbeitszeiten, körperliche Belastungen am Arbeitsplatz und eine als sinnvoll wahrgenommene Tätigkeit identifiziert. Ebenfalls signifikante Einflüsse zeigen sich für die Aufstiegschancen am Arbeitsplatz, die wahrgenommene Sicherheit des Arbeitsplatzes und die persönlichen Beziehungen zu Kollegen und Vorgesetzten. Nach Kontrolle für diese Faktoren ist das Niveau der Arbeitszufriedenheit in den skandinavischen Staaten signifikant höher als in allen anderen Länderclustern. Der größte Rückstand ergibt sich auch hierbei für die Türkei.

Um zu prüfen, ob die Bestimmungsfaktoren der Arbeitszufriedenheit abhängig vom Wohlstandsniveau eines Landes und der vorhandenen sozialen Absicherung sind, werden Ordered-Probit Regressionsmodelle getrennt für fünf Ländergruppen geschätzt. Dabei zeigen sich sowohl für unterschiedliche Wohlfahrtsniveaus als auch für Staaten mit unterschiedlichem Niveau sozialer Absicherung erhebliche Unterschiede. Während in Ländern mit hohem Wohlstand und starker Absicherung der Inhalt der Tätigkeit den größten Einfluss ausübt und auch die Autonomie bei der Erledigung der Arbeitsaufgaben eine Rolle spielt, ist die subjektive Einschätzung gut bezahlt zu werden, in allen anderen Ländern dominierend. Bezüglich der Arbeitszeiten zeigt sich in Ländern mit niedrigerem Wohlstandsniveau ein geringerer negativer Einfluss, wenn Arbeitszeiten mit privaten Verpflichtungen kollidieren.

Der insgesamt starke Einfluss der subjektiven Einkommensbewertung steht in Widerspruch zu Ergebnissen von Sousa-Poza und Sousa-Poza (2000), die diesen Effekt nicht für alle Länder finden, sowie zu Clark (2005), der das Einkommen als weniger wichtige Determinante der Arbeitszufriedenheit erachtet.

\section{Überblick über die wichtigsten Ergebnisse unserer Studie:}

Die Arbeitszufriedenheit ist besonders hoch in den skandinavischen Staaten, Großbritannien und Irland, niedrig hingegen in Osteuropa, Griechenland und der Türkei.

Auf Makroebene über alle 31 europäischen Länder korreliert die Arbeitszufriedenheit am stärksten mit dem Arbeitsumfang, dem Einkommen und körperlichen Belastungen durch die Arbeit.

Bei einer multivariaten Ordered-Probit Analyse gemeinsam für alle 31 Staaten ergeben sich als entscheidende Einflussfaktoren: Der Lohnsatz, die subjektive Einschätzung gut bezahlt zu 
sein, die Ausgestaltung der Arbeitszeiten, körperliche Belastungen am Arbeitsplatz und eine als sinnvoll wahrgenommene Tätigkeit.

Nach Kontrolle für diese Faktoren ist das Niveau der Arbeitszufriedenheit in den skandinavischen Staaten signifikant höher als in allen anderen Länderclustern. Der größte Rückstand ergibt sich für die Türkei.

Eine Ordered-Probit Analyse getrennt für fünf Ländergruppen mit unterschiedlich ausgeprägten Wohlstandsniveaus, Wohlfahrtsstaaten und Arbeitsbedingungen zeigt:

Während in Skandinavien und Kontinentaleuropa die Einschätzung, einer sinnvollen Tätigkeit nachzugehen, den größten Einfluss ausübt, ist die subjektive Einschätzung, gut bezahlt zu werden, in allen anderen Ländern dominierend.

Bezüglich der Arbeitszeiten zeigt sich in Ländern mit niedrigerem Wohlstandsniveau ein geringerer negativer Einfluss, wenn Arbeitszeiten mit privaten Verpflichtungen kollidieren.

In Großbritannien und Irland spielt die Sicherheit des Arbeitsplatzes eine größere Rolle als im Rest der betrachteten Länder.

Die Autonomie bei der Organisation der Arbeitsaufgaben findet sich nur in Staaten mit hohem Wohlstandsniveau (Großbritannien, Irland, Kontinentaleuropa und Skandinavien) unter den zehn stärksten Einflussfaktoren.

Wie gezeigt, ermöglicht die Analyse einer auf europäischer Ebene in einheitlichem Design erhobenen Datenquelle, dem EWCS, die vergleichende Untersuchung von Erklärungsmustern der Arbeitszufriedenheit in Staaten mit unterschiedlichen Wohlfahrtsregimes, Wohlstandsniveaus und Arbeitsbedingungen. Unsere Ergebnisse betonen dabei, dass für einen internationalen Vergleich der Arbeitszufriedenheit eine Berücksichtigung national unterschiedlicher Erklärungsmuster notwendig erscheint.

Für eine weitergehende Analyse divergierender Erklärungsmuster auf nationaler Ebene wären international vergleichbare Paneldaten mit detaillierten Informationen über die Arbeitsbedingungen und Arbeitszeitarrangements von Nutzen. Dies würde die Berücksichtigung individueller fixer Effekte (z.B. Persönlichkeitsmerkmale) ermöglichen. Diese können das Antwortverhalten auf subjektive Wohlfahrtsmaße sowie auch das Arbeitsmarktverhalten beeinflussen und können bei Nichtberücksichtigung zu einer Verzerrung der Ergebnisse führen. 


\section{Anhang}

Tabelle 8: Verteilung der Arbeitszufriedenheit in den 31 EWCS Staaten

\begin{tabular}{|c|c|c|c|c|c|c|}
\hline Land & $\varnothing$ & $\begin{array}{c}\text { Standard- } \\
\text { abweichung }\end{array}$ & -- & - & + & ++ \\
\hline Dänemark & 3.39 & 0.68 & 2.04 & 4.88 & 44.89 & 48.19 \\
\hline Großbritannien & 3.37 & 0.66 & 1.48 & 5.87 & 47.08 & 45.57 \\
\hline Norwegen & 3.30 & 0.64 & 1.25 & 6.02 & 54.37 & 38.36 \\
\hline Schweiz & 3.27 & 0.65 & 0.98 & 8.13 & 54.05 & 36.84 \\
\hline Österreich & 3.26 & 0.69 & 1.96 & 8.33 & 51.28 & 38.43 \\
\hline Zypern & 3.23 & 0.78 & 2.83 & 13.18 & 41.84 & 42.14 \\
\hline Belgien & 3.21 & 0.68 & 1.93 & 8.96 & 55.31 & 33.81 \\
\hline Irland & 3.15 & 0.71 & 2.62 & 11.16 & 54.69 & 31.53 \\
\hline Deutschland & 3.14 & 0.63 & 1.45 & 9.36 & 63.06 & 26.13 \\
\hline Niederlande & 3.09 & 0.66 & 3.37 & 7.62 & 65.20 & 23.80 \\
\hline Luxemburg & 3.09 & 0.72 & 3.82 & 9.96 & 59.36 & 26.87 \\
\hline Schweden & 3.09 & 0.69 & 2.34 & 12.36 & 59.13 & 26.17 \\
\hline Malta & 3.06 & 0.74 & 3.32 & 14.53 & 55.42 & 26.74 \\
\hline Frankreich & 3.04 & 0.74 & 3.89 & 13.53 & 57.25 & 25.32 \\
\hline Finnland & 3.04 & 0.66 & 2.22 & 13.43 & 62.97 & 21.39 \\
\hline Portugal & 3.02 & 0.67 & 2.97 & 12.30 & 64.69 & 20.04 \\
\hline EWCS31 Ø & 3.00 & 0.76 & 4.63 & 15.09 & 56.11 & 24.16 \\
\hline Tschechien & 2.95 & 0.62 & 0.82 & 19.44 & 63.86 & 15.88 \\
\hline Spanien & 2.92 & 0.72 & 4.42 & 17.33 & 60.33 & 17.91 \\
\hline Polen & 2.90 & 0.69 & 4.12 & 17.08 & 63.83 & 14.97 \\
\hline Kroatien & 2.89 & 0.83 & 6.09 & 21.62 & 49.08 & 23.21 \\
\hline Italien & 2.88 & 0.75 & 4.73 & 20.63 & 56.61 & 18.03 \\
\hline Slowakei & 2.88 & 0.70 & 3.45 & 21.04 & 59.88 & 15.63 \\
\hline Estland & 2.82 & 0.71 & 5.08 & 20.24 & 62.16 & 12.52 \\
\hline Ungarn & 2.82 & 0.70 & 5.42 & 18.80 & 64.50 & 11.29 \\
\hline Slowenien & 2.79 & 0.71 & 5.02 & 23.26 & 59.68 & 12.04 \\
\hline Litauen & 2.77 & 0.72 & 3.80 & 29.16 & 53.61 & 13.44 \\
\hline Lettland & 2.76 & 0.69 & 4.63 & 25.10 & 60.27 & 10.00 \\
\hline Bulgarien & 2.73 & 0.80 & 7.99 & 25.24 & 52.32 & 14.45 \\
\hline
\end{tabular}




\begin{tabular}{lcccccc}
\hline Land & $\varnothing$ & $\begin{array}{c}\text { Standard- } \\
\text { abweichung }\end{array}$ & -- & - & + & ++ \\
\hline Griechenland & 2.69 & 0.93 & 12.13 & 27.04 & 40.57 & 20.25 \\
Rumänien & 2.65 & 0.82 & 9.25 & 29.20 & 48.54 & 13.01 \\
Türkei & 2.47 & 0.90 & 17.73 & 28.10 & 43.67 & 10.50 \\
\hline
\end{tabular}

Hinweis: --/-/+/++ entsprechen den Fragebogenformulierungen überhaupt nicht zufrieden/ nicht sehr zufrieden/ zufrieden/sehr zufrieden.

Quelle: Eigene Berechnungen basierend auf den Daten des EWCS 2005, gewichtet (w4).

\section{Tabelle 9: Variablendefinitionen der Arbeitsplatzcharakteristika}

\begin{tabular}{|c|c|}
\hline Variable & Definition [Kontrast bei Berechnung von $\Delta \mathrm{P}(\mathrm{Y}=4)$ ] \\
\hline $\ln ($ Lohnsatz) & $\begin{array}{l}\text { = Nettoeinkommen in } € \text { aus Hauptbeschäftigung / übliche Wochenstunden } \\
\text { (Einkommen wurde in zehn Einkommensklassen erfasst; für jede Klasse } \\
\text { wurde der Mittelwert eingesetzt; Ausnahmen: unterste Klasse: } \\
0,75^{*} \text { Untergrenze, oberste Klasse: } 1,2 * \text { Untergrenze; alle Währungen zum } \\
\text { Wechselkurs des } 20 \text {. Oktober 2005) [Kontrast: } 1 \text {. Dezil vs. 9. Dezil] }\end{array}$ \\
\hline Subjektiv gut bezahlt & $=$ ich werde für meine Arbeit gut bezahlt: Stimme stark zu; stimme zu \\
\hline 1. Quintil & $=$ Nettoeinkommen liegt auf nationaler Ebene im 1. Quintil (gewichtet w4) \\
\hline Arbeitsstunden/Woche & $=$ übliche Wochenarbeitsstunden [Kontrast 35 gegen 45 Stunden] \\
\hline $\begin{array}{l}\text { Flexibilität für Arbeitgeber } \\
\text { (Index) }\end{array}$ & $\begin{array}{l}\text { = Anteil zutreffend von fünf (Schichtarbeit; wöchentlich variierende Ar- } \\
\text { beitstage; variierende tägliche Arbeitszeiten; Arbeitszeiten ändern sich mit } \\
\text { wenigen Tagen oder weniger Vorlauf; mindestens mehrmals pro Monat } \\
\text { außerhalb der Arbeitszeiten kontaktiert) [Kontrast } 0 \text { gegen 1] }\end{array}$ \\
\hline $\begin{array}{l}\text { Flexibilität für Arbeit- } \\
\text { nehmer } \\
\text { (Index) }\end{array}$ & $\begin{array}{l}\text { = Anteil zutreffend von drei (Sie können frei entscheiden wann sie Urlaub } \\
\text { oder Tage frei nehmen - fast immer/häufig; Sie können ihre Pausen ma- } \\
\text { chen wann sie möchten - fast immer/häufig; Man kann die Arbeitszeit } \\
\text { innerhalb eines gewissen Rahmens anpassen (z.B. Gleitzeit) oder Die Ar- } \\
\text { beitszeit kann vollständig individuell festgelegt werden) [Kontrast } 0 \text { gegen } \\
\text { 1] }\end{array}$ \\
\hline $\begin{array}{l}\text { AZ außer Norm } \\
\text { (Index) }\end{array}$ & $\begin{array}{l}\text { = Anteil zutreffend von vier (mind. ein Samstag/Monat; mind. ein Sonn- } \\
\text { tag/Monat; mind. vier Abende/Monat; mind. vier Nächte/Monat); [Kontrast } \\
0 \text { gegen 1] }\end{array}$ \\
\hline $\begin{array}{l}\text { Arbeitszeit (AZ)-privat } \\
\text { Konflikt }\end{array}$ & $\begin{array}{l}=\text { Wie gut lassen sich Ihre Arbeitszeiten im Allgemeinen mit Ihren familiä- } \\
\text { ren oder sozialen Verpflichtungen außerhalb des Berufs vereinbaren? - } \\
\text { 1=nicht sehr gut/ überhaupt nicht gut }\end{array}$ \\
\hline $\begin{array}{l}\text { Körperliche Belastung } \\
\text { (Index) }\end{array}$ & $\begin{array}{l}\text { = Anteil zutreffend von zehn - Inwieweit sind Sie bei der Arbeit den fol- } \\
\text { genden Bedingungen ausgesetzt - mind. ein Viertel der Zeit (Vibrationen } \\
\text { von Werkzeugen, Maschinen usw.; starker Lärm...; hohe Temperaturen...; } \\
\text { niedrige Temperaturen...; Einatmen von Rauch, Dämpfen...; Einatmen } \\
\text { von Dämpfen, z.B. Lösungsmittel...; Umgang oder Hautkontakt mit che- } \\
\text { mischen Produkten oder Substanzen; Strahlung...; Tabakrauch von ande- } \\
\text { ren Personen; Umgang oder direkter Kontakt mit eventuell ansteckenden } \\
\text { Stoffen wie Abfall, Körperflüssigkeiten, Labormaterialien usw.) [Kontrast } \\
0 \text { gegen 0,5] }\end{array}$ \\
\hline $\begin{array}{l}\text { Autonomie } \\
\text { (Index) }\end{array}$ & $\begin{array}{l}=\text { Anteil zutreffend von drei (Können Sie sich folgendes aussuchen bzw. } \\
\text { ändern oder nicht? (die Reihenfolge Ihrer Aufgaben; das Vorgehen bei der } \\
\text { Erledigung einer Aufgabe; Ihr Arbeitstempo oder Ihren Arbeitsrhythmus) } \\
\text { [Kontrast } 0 \text { gegen 1] }\end{array}$ \\
\hline Vorgesetzter & $=$ Vorgesetzter für mind. eine Person \\
\hline
\end{tabular}




\begin{tabular}{|c|c|}
\hline Variable & Definition [Kontrast bei Berechnung von $\Delta P(Y=4)]$ \\
\hline $\begin{array}{l}\text { Anspruchsvoll } \\
\text { (Index) }\end{array}$ & $\begin{array}{l}\text { = Anteil zutreffend von vier (Erfordern die Aufgaben verschiedene Fähig- } \\
\text { keiten/Fertigkeiten; Wechseln Sie sich bei bestimmten Aufgaben mit Ihren } \\
\text { Kollegen ab?; Schließt die Arbeit, die Sie hauptsächlich ausüben, im All- } \\
\text { gemeinen folgendes ein?... Komplexe Arbeitsaufgaben; Neues lernen) } \\
\text { [Kontrast } 0 \text { gegen 1] }\end{array}$ \\
\hline $\begin{array}{l}\text { Sinn } \\
\text { (Index) }\end{array}$ & $\begin{array}{l}=\text { Anteil zutreffend von zwei (Sie haben das Gefühl eine sinnvolle Arbeit } \\
\text { zu verrichten; Ihre Tätigkeit gibt Ihnen das Gefühl gute Arbeit getan zu } \\
\text { haben) [Kontrast } 0 \text { gegen 1] }\end{array}$ \\
\hline Aufstiegsmöglichkeiten & $=$ Meine Arbeit bietet gute Karrierechancen (Stimme stark zu; stimme zu) \\
\hline Befristet & $=$ zeitlich befristete Beschäftigung \\
\hline Arbeitsplatz (AP) gefährdet & $\begin{array}{l}\text { = Ich könnte meine Arbeit in den nächsten sechs Monaten verlieren (Stim- } \\
\text { me stark zu; stimme zu) }\end{array}$ \\
\hline Pers. Beziehungen (Index) & $\begin{array}{l}\text { = Anteil zutreffend von drei (Sie können Hilfe von Kollegen bekommen, } \\
\text { wenn Sie darum bitten (fast immer, häufig, manchmal); bei Anfrage wer- } \\
\text { den Sie von Ihrem Vorgesetzten/Ihrem Chef unterstützt ; Ich habe sehr gute } \\
\text { Freunde bei der Arbeit (Stimme stark zu; stimme zu)) [Kontrast } 0 \text { gegen 1] }\end{array}$ \\
\hline
\end{tabular}

\section{Tabelle 10: Übersicht der erklärenden Größen in der multivariaten Analyse}

\begin{tabular}{|c|c|}
\hline Personenvariablen & höchster Bildungsabschluss (Ref.: no or primary) \\
\hline Mann & - lower secondary \\
\hline Altersgruppen (Ref.: 60-64) & - upper secondary \\
\hline$-15-19$ & - post secondary incl. vocational education \\
\hline$-20-24$ & - tertiary education \\
\hline$-25-29$ & Berufserfahrung in Jahren \\
\hline$-30-34$ & Berufserfahrung ${ }^{2} / 100$ \\
\hline$-35-39$ & Krankheitstage im letzten Jahr (Ref.: 0 Tage) \\
\hline$-40-44$ & $-1-10$ \\
\hline$-45-49$ & $-11-20$ \\
\hline$-50-54$ & $-21-50$ \\
\hline$-55-59$ & $-51-365$ \\
\hline Haushaltsvariablen & HH mit zwei oder mehr Erwerbstätigen \\
\hline Haushaltsgröße (Ref.: Single-Haushalt) & Kinder jünger als 16 im HH (Ref.: kein Kind) \\
\hline-2 & -1 \\
\hline-3 & -2 \\
\hline-4 & -3 oder mehr \\
\hline-5 & Hauptverdiener im HH (Ref.: Ja) \\
\hline-6 oder mehr & - etwa gleich verteilt \\
\hline Leben in einer Partnerschaft & - nein \\
\hline
\end{tabular}




\begin{tabular}{ll}
$\begin{array}{l}\text { Arbeitsverhältnis } \\
\text { Betriebszugehörigkeit in Jahren }\end{array}$ & $\begin{array}{l}\text { Sorgen um Arbeitsplatz (6 Monate) } \\
\text { Betriebszugehörigkeit }{ }^{2} / 100\end{array}$ \\
befristete Anstellung & Arbeitsweg ${ }^{2} / 100$ \\
\hline Arbeitszeiten & \\
Arbeitsstunden pro Woche & Flexibilität für Arbeitgeber \\
Arbeitsstunden pro Woche $/ 100$ & Flexibilität für Arbeitnehmer \\
AZ kollidiert mit privaten Verpflichtungen & Ungünstige Arbeitszeiten \\
\hline Tätigkeit körperlich/geistige Anforderungen & Anspruchsvolle Tätigkeit \\
Index körperliche Belastung & sinnvolle Tätigkeit / gute Arbeit \\
Autonomieindex & Branchen (Ref. +11) \\
Vorgesetzter & Tätigkeit (Ref. + 9) \\
\hline Einkommen & subjektiv Gut bezahlt \\
ln(Lohnsatz) & 1. Quintil \\
\hline
\end{tabular}

\section{Karrieremöglichkeiten}

Karriereaussicht gut

Persönliche Beziehungen

gute persönliche Beziehungen

Quelle: Eigene Zusammenstellung.

Tabelle 11: Marginale Effekte auf $P(Y=4)$ für Bonoli+1 Cluster

\begin{tabular}{lrlrlrc}
\hline UK und Irland & & Kontinentaleuropa & & \multicolumn{2}{l}{ Skandinavien } \\
\hline subjektiv gut bezahlt & 22.90 & Sinn & 17.35 & Sinn & 27.59 \\
Arbeitszeit/privat-Konflikt & -16.41 & subjektiv gut bezahlt & 15.54 & Arbeitszeit/privat-Konflikt & -15.65 \\
Arbeitsplatz unsicher & -15.97 & Arbeitszeit/privat-Konflikt & -14.69 & subjektiv gut bezahlt & 14.29 \\
Sinn & 15.40 & Aufstiegsmöglichkeiten & 12.49 & ln(Lohnsatz) & 11.99 \\
Aufstiegsmöglichkeiten & 12.75 & körperliche Belastung & -9.62 & körperliche Belastung & -11.91 \\
Flex. für Arbeitnehmer & 12.17 & Flex. für Arbeitnehmer & 8.91 & Aufstiegsmöglichkeiten & 11.26 \\
persönliche Beziehungen & 11.34 & Arbeitsplatz unsicher & -8.32 & Flex. für Arbeitnehmer & 10.41 \\
Autonomie & 10.81 & ln(Lohnsatz) & -8.04 & persönliche Beziehungen & 9.01 \\
körperliche Belastung & -8.77 & persönliche Beziehungen & 7.43 & Autonomie & 8.68 \\
Vorgesetzter & 7.86 & Autonomie & 5.05 & Arbeitsplatz unsicher & -8.56 \\
\hline
\end{tabular}




\begin{tabular}{lrlr}
\hline Südeuropa & \multicolumn{3}{l}{ Osteuropa + Türkei } \\
\hline subjektiv gut bezahlt & 16.21 & subjektiv gut bezahlt & 11.88 \\
Sinn & 13.58 & Sinn & 7.41 \\
Aufstiegsmöglichkeiten & 11.55 & Aufstiegsmöglichkeiten & 7.34 \\
Arbeitszeit/privat-Konflikt & -9.34 & Arbeitszeit/privat-Konflikt & -5.66 \\
körperliche Belastung & -8.82 & körperliche Belastung & -5.42 \\
Flex. für Arbeitnehmer & 7.85 & Flex. für Arbeitnehmer & 5.10 \\
Arbeitsplatz unsicher & -7.84 & ln(Lohnsatz) & 4.83 \\
persönliche Beziehungen & 5.64 & persönliche Beziehungen & 3.81 \\
Vorgesetzter & 4.74 & Arbeitsplatz unsicher & -3.42 \\
befristet & 3.03 & Flex. für Arbeitgeber & -2.20 \\
\hline
\end{tabular}

Hinweis: Detaillierte Informationen zur Definition der dargestellten Arbeitsmarktcharakteristika finden sich in Tabelle 9 oben. Die Berechnung der marginalen Effekte erfolgte für eine hypothetische Person, die bei allen Merkmalen die Durchschnittswerte des jeweiligen Clusters aufweist.

Quelle: Eigene Berechnungen basierend auf EWCS 2005. 


\section{Literaturverzeichnis}

Ahn N. and J.R. García (2004): Job Satisfaction in Europe, FEDEA Discussion Paper Series, No. 2004-016.

Akerlof G.A., Rose A.K. and J.L. Yellen (1988): Job switching and job satisfaction in the US labor market, In: Brookings papers on economic activity, 495-582.

Arts W. and J. Gelissen (2002): Three worlds of welfare capitalism or more? - A state-of-the-artreport, In: Journal of European Social Policy, Vol. 12, No. 2, 137-158.

Bender K.A., Donohue S.M. and J.S. Heywood (2005): Job satisfaction and gender segregation, In: Oxford Economic Papers, Vol. 57, No. 3, 479-496.

Bonoli G. (1997): Classifying welfare states - A two-dimension approach, In: Journal of Social Policy, Vol. 26, 351-372.

Blanchflower D.G. and A.J. Oswald (1999): Well-being, insecurity and the decline of American job satisfaction, University of Warwick.

Clark A.E. and A.J. Oswald (1996): Satisfaction and comparison income, In: Journal of public economics, Vol. 61, No. 3, 359-381.

Clark A.E. (1997): Job satisfaction and gender - Why are women so happy at work?, In: Labour economics, Vol. 4, No. 4, 341-372.

Clark A.E. (2001): What really matters in a job? - Hedonic measurement using quit data, In: Labour economics, Vol. 8, No. 2, 223-242.

Clark A.E. (2005): What makes a good job? - Evidence from OECD countries, In: Bazen, S., Lucifora, C. and W. Salverda (2005): Job quality and employer behaviour, Palgrave Macmillan, Houndmills, Basingstoke, Hampshire.

D'Addio A.C., Eriksson T. and P. Frijters (2007): An analysis of the determinants of job satisfaction when individuals' baseline satisfaction levels may differ, In: Applied economics, Vol. 39, $2413-2423$.

Diener E., Diener M. and C. Diener (1995): Factors predicting the subjective well-being of nations, In: Journal of Personality and Social Psychology, Vol. 69, No. 5, 851-864.

Easterlin R.A. (1974): Does economic growth improve the human lot? - Some empirical evidence, In: David, P.A., Reder, M.W. and M. Abramovitz (1974): Nations and households in economic growth - Essays in honor of Moses Abramovitz, Academic Press, New York, 89-125.

Esping-Andersen G. (1990): The three worlds of welfare capitalism, Polity Press, Cambridge.

Eurobarometer (2009): Eurobarometer 72 - Public Opinion in the European Union, Brüssel.

European Foundation for the Improvement of Living and Working Conditions (2007): European Working Conditions Survey 2005 [computer file], UK Data Archive, Colchester, Essex.

Fenger H.J.M. (2007): Welfare regimes in Central and Eastern Europe - Incorporating postcommunist countries in a welfare regime typology, In: Contemporary Issues and Ideas in Social Sciences, Vol. 3, No. 2, 1-30.

Ferrera M. (1996): The "southern" model of welfare in social Europe, In: Journal of European Social Policy, Vol. 6, No. 1, 17-37.

Freeman R.B. (1978): Job satisfaction as an economic variable, In: The American economic review, Vol. 68, No. 2, 135-141.

Frey B.S. (1997): Not just for the money - An economic theory of personal motivation, Elgar, Cheltenham. 
Hamermesh D.S. (2001): The Changing Distribution of Job Satisfaction, In: The Journal of Human Resources, Vol. 36, No. 1, 1-30.

Hamermesh D.S. (2004): Subjective Outcomes in Economics - Association Lecture, In: Southern Economic Journal, Vol. 71, No. 1, 2-11.

Hanglberger D. (2010): Arbeitszufriedenheit und flexible Arbeitszeiten - Empirische Analyse mit Daten des Sozio-ökonomischen Panels, FFB-Diskussionspapiere, No. 80, Lüneburg.

Kaiser L.C. (2007): Gender-job satisfaction differences across Europe: An indicator for labour market modernization, In: International Journal of Manpower, Vol. 28, No. 1, 75-94.

Koys D.J. (2001): The effects of employee satisfaction, organizational citizenship behavior, and turnover on organizational effectiveness - A unit-level, longitudinal study, In: Personnel Psychology, Vol. 54, No. 1, 101-114.

Kristensen N. and E. Johansson (2008): New Evidence on Cross-Country Differences in Job Satisfaction Using Anchoring Vignettes, In: Labour economics, Vol. 15, No. 1, 96-117.

Layard R. (2003): Happiness - Has social science a clue?, London School of Economics, London.

Long J.S. (1997): Regression models for categorical and limited dependent variables, Sage Publications, Thousand Oaks, California.

Martin A. (2002): Arbeitsbedingungen von Selbständigen, Schriften aus dem Institut für Mittelstandsforschung, No. 18, Lüneburg.

McKelvey R.D. and W. Zavoina (1971): An IBM Fortran IV Program to Perform N-Chotomus Multivariate Probit Analysis, In: Behavioral Science, Vol. 16, No. 2, 186-187.

McKelvey R.D. and W. Zavoina (1975): A Statistical Model for the Analysis of Ordinal Level Dependent Variables, In: Journal of Mathematical Sociology, Vol. 4, 103-120.

Merz, J. (Hg.) (2008): Freie Berufe - Einkommen und Steuern (FB€St) - Beiträge aus Wissenschaft, Praxis und Politik, Nomos Verlagsgesellschaft, Baden-Baden.

Merz J. and P. Böhm (2005): Arbeitszeitarrangements und Entlohnung - Ein Treatment-Effects-Ansatz für Freiberufler, Unternehmer und abhängig Beschäftigte, In: Schulte, R. (2005): Ergebnisse der MittelstandsForschung, Lit-Verlag, Münster, 237-264.

Merz J. and P. Böhm (2008): Tägliche Arbeitszeitmuster und Einkommen von Freien Berufen - Neue Ergebnisse auf der Basis von Zeittagebüchern, In: Merz, J. (2008): Freie Berufe - Einkommen und Steuern (FB€St) - Beiträge aus Wissenschaft, Praxis und Politik, Nomos Verlagsgesellschaft, Baden-Baden, 93-118.

Merz J. and D. Burgert (2003): Working Hour Arrangements and Working Hours - A Microeconometric Analysis Based on German Time Diary Data, FFB-Diskussionspapiere, No. 41, Lüneburg.

Merz J. and D. Burgert (2004a): Wer arbeitet wann? Arbeitszeitarrangements von Selbständigen und abhängig Beschäftigten - Eine mikroökonometrische Analyse deutscher Zeitbudgetdaten, FFB-Diskussionspapiere, No. 45, Lüneburg.

Merz J. and D. Burgert (2004b): Arbeitszeitarrangements - Neue Ergebnisse aus der Zeitbudgeterhebung 2001/02 im Zeitvergleich zu 1991/92, In: Schäfer, D. (2004): Alltag in Deutschland Analysen zur Zeitverwendung, Statistisches Bundesamt, Wiesbaden, 304-336.

Merz J., Böhm P. and D. Burgert (2009): Timing and Fragmentation of Daily Working Hours Arrangements and Income Inequality - An Earnings Treatment Effects Approach with German Time Use Diary Data, In: electronic International Journal of Time Use Research, Vol. 6, No. 2, 200-239.

Merz, J. and M. Ehling (Hg.) (1999): Time use - Research, data and policy, Nomos Verlagsgesellschaft, Baden-Baden. 
Merz J. and R. Lang (1999): Working hour tension dynamics - Professions, entrepreneurs and employees in a ten years panelanalysis of desired vs. actual working hours incorporating nonmarket influences, In: Merz, J. and M. Ehling (1999): Time use - Research, data and policy, Nomos Verlagsgesellschaft, Baden-Baden, 241-268.

Nicoletti C. (2006): Differences in job dissatisfaction across Europe, ISER Working Paper, No. 200642, Cambridge.

Origo F. and L. Pagani (2006): Is work flexibility a stairway to heaven? - The story told by job satisfaction in Europe, University of Milan Working Paper Series, No. 97, Milan.

Parent-Thirion A., Fernández Macías E., Hurley J. and G. Vermeylen (2007): Fourth European Working Conditions Survey, Office for Official Publications of the European Communities.

Patterson M.G., West M.A., Lawthom R. and S. Nickell (1997): Impact of people management strategies on Business Performance, Institute of Personnel and Development, Issues in People Management, London.

Patterson M., Warr P. and M. West (2004): Organizational climate and company productivity - The role of employee affect and employee level, In: Journal of Occupational and Organizational Psychology, Vol. 77, No. 2, 193-216.

Pfeffer J. and N. Langton (1993): The Effect of Wage Dispersion on Satisfaction, Productivity, and Working Collaboratively - Evidence from College and University Faculty, In: Administrative Science Quarterly, Vol. 38, No. 3, 382-407.

Rhein T. (2010): Beschäftigungsdynamik im internationalen Vergleich - Ist Europa auf dem Weg zum "Turbo-Arbeitsmarkt"?, IAB, IAB-Kurzbericht, Nürnberg.

Salancik G.R. and J. Pfeffer (1977): An Examination of Need-Satisfaction Models of Job Attitudes, In: Administrative Science Quaterly, Vol. 22, No. 3, 427-456.

Schäfer, D. (Hg.) (2004): Alltag in Deutschland - Analysen zur Zeitverwendung, Statistisches Bundesamt, Wiesbaden.

Schulte, R. (Hg.) (2005): Ergebnisse der MittelstandsForschung, Lit-Verlag, Münster.

Sharkh M.A. and I. Gough (2010): Global welfare regimes - A cluster analysis, In: Global Social Policy, Vol. 10, No. 1, 27-58.

Sousa-Poza A. and A.A. Sousa-Poza (2000): Well-being at work - A cross-national analysis of the levels and determinants of job satisfaction, In: The journal of socio-economics, Vol. 29, No. 6, 517-538.

Stiglitz J.E., Sen A. and J.-P. Fitoussi (2009): Report by the Commission on the Measurement of Economic Performance and Social Progress, http://www.stiglitz-senfitoussi.fr/documents/rapport_anglais.pdf, zuletzt geprüft am 22.09.2010.

Veenhoven R. (2008): Comparability of happiness across nations, In: School of Sociology and Social Work Journal, No. 104, 211-234.

Warr P. (1999): Well-Being at the workplace, In: Diener, E., Kahneman, D. and N. Schwarz (1999): Well-being - The foundations of hedonic psychology, Russell Sage Foundation, New York, 392-412. 
Forschungsinstitut Freie Berufe (FFB), Universität Lüneburg Publikationen

FFB-Jahresberichte

FFB-Bücher in der FFB-Schriftenreihe

FFB-Bücher

FFB-Gutachten

FFB-Artikel in wissenschaftlichen Zeitschriften und Büchern

FFB-Reprints

FFB-Dokumentationen, ISSN 1615-0376

FFB Sonstige Arbeitsberichte, ISSN 0175-7275

www.leuphana.de/ffb

\section{FFB-Diskussionspapiere, ISSN 0942-2595}

Merz, J. und Henning Stolze, D., 2010, Kumulation von Querschnitten - Evaluierung alternativer Konzepte kumulierten laufenden Wirtschaftsrechnungen bis 2003 im Vergleich zur EinkommensVerbrauchsstichprobe 2003, FFB-Discussionpaper No. 85, Fakultät II, Wirtschafts- Verhaltens- und Rechtswissenschaften, Leuphana Universität Lüneburg, Lüneburg.

FFB-DP Nr.85

Merz, J. und Henning Stolze, D., 2010, Cumulation of Cross-Section Surveys - Evaluation of Alternative Concepts for the Cumulated Continuous Household Budget Surveys (LWR) 1999 until 2003 compared to the Sample Survey of Income and Expenditures (EVS) 2003, FFB-Discussionpaper No. 84, Fakultät II, Wirtschafts- Verhaltens- und Rechtswissenschaften, Leuphana Universität Lüneburg, Lüneburg.

FFB-DP Nr.84

Österbacka, E., Merz, J. and C.D. Zick., 2010, Human Capital Investments in Children: A Comparative Analysis of the Role of Parent-Child Shared Time in Selected Countries, FFB-Diskussionspapier Nr. 83, Fakultät II, Wirtschafts-, Verhaltens- und Rechtswissenschaften, Leuphana Universität Lüneburg, Lüneburg.

FFB-DP Nr. 83

Merz, J. und T. Rathjen, 2010, Sind Selbständige zeit- und einkommensarm? Eine Mikroanalyse der Dynamik interdependenter multidimensionaler Armut mit dem Sozio-ökonomischen Panel und den deutschen Zeitbudgeterhebungen, FFB-Diskussionspapier Nr. 82, Fakultät II, Wirtschafts-, Verhaltens- und Rechtswissenschaften, Leuphana Universität Lüneburg, Lüneburg.

FFB-DP Nr. 82

Rucha, R., 2010, Effekte einer freiwilligen Mitgliedschaft in Berufsverbänden auf das Einkommen - Eine Analyse der Einkommensdynamik bei Freiberuflern in Deutschland, FFB-Diskussionspapier Nr. 81, Fakultät II, Wirtschafts-, Verhaltens- und Rechtswissenschaften, Leuphana Universität Lüneburg, Lüneburg.

FFB-DP Nr. 81

Hanglberger, D., 2010, Arbeitszufriedenheit und flexible Arbeitszeiten - Empirische Analyse mit Daten des Sozio-oekonomischen Panels, FFB-Diskussionspapier Nr. 80, Fakultät II, Wirtschafts-, Verhaltens- und Rechtswissenschaften, Leuphana Universität Lüneburg, Lüneburg.

FFB-DP Nr. 80

Merz, J. and T. Rathjen, 2009, Time and Income Poverty - An Interdependent Multidimensional Poverty Approach with German Time Use Diary Data, FFB-Discussionpaper No. 79, Fakultät II, Wirtschafts-, Verhaltens- und Rechtswissenschaften, Leuphana Universität Lüneburg, Lüneburg.

FFB-DP Nr. 79

Merz, J., 2009, Time Use and Time Budgets - Improvements, Future Challenges and Recommendations, FFBDiscussionpaper, No. 78, Fakultät II, Wirtschafts-, Verhaltens- und Rechtswissenschaften, Leuphana Universität Lüneburg, Lüneburg.

FFB-DP Nr. 78 
Merz, J., Hanglberger, D. and R. Rucha, 2009, The Timing of Daily Demand for Goods and Services Multivariate Probit Estimates and Microsimulation Results for an Aged Population with German Time Use Diary Data, FFB-Discussionpaper No. 77, Fakultät II, Wirtschafts-, Verhaltens- und Rechtswissenschaften, Leuphana Universität Lüneburg, Lüneburg.

FFB-DP Nr. 77

Merz, J., 2009, Zeitverwendungsforschung und Mediennutzung, FFB-Diskussionspapier Nr. 75, Fakultät II, Wirtschafts-, Verhaltens- und Rechtswissenschaften, Universität Lüneburg, Lüneburg. FFB-DP Nr. 76

Böhm, P. und. J. Merz, 2008, Reichtum in Niedersachsen und anderen Bundesländern - Ergebnisse aus der Steuer-Geschäftsstatistik 2003 für Selbständige (Freie Berufe und Unternehmer) und abhängig Beschäftigte, FFB-Diskussionspapier Nr. 75, Fakultät II, Wirtschafts-, Verhaltens- und Rechtswissenschaften, Universität Lüneburg, Lüneburg.

FFB-DP Nr. 75

Merz, J. und M. Zwick, 2008, Einkommens- und Verteilungsanalysen mit dem Taxpayer-Panel - Neue Möglichkeiten und erste Ergebnisse für Selbständige als Freiberufler und Unternehmer und abhängig Beschäftigte sowie für hohe Einkommen, FFB-Diskussionspapier Nr. 74, Fakultät II, Wirtschafts-, Verhaltens- und Rechtswissenschaften, Universität Lüneburg, Lüneburg.

FFB-DP Nr. 74

Böhm, P. und J. Merz, 2008, Zum Einkommensreichtum Älterer in Deutschland - Neue Reichtumskennzahlen und Ergebnisse aus der Lohn- und Einkommenssteuerstatistik (FAST 2001), FFB-Diskussionspapier Nr. 73, Fakultät II, Wirtschafts-, Verhaltens- und Rechtswissenschaften, Universität Lüneburg, Lüneburg.

FFB-DP Nr. 73

Herrmann, H., 2007, Freie Berufe - Europäische Entwicklungen, FFB-Diskussionspapier Nr. 72, Fakultät II, Wirtschafts-, Verhaltens- und Rechtswissenschaften, Universität Lüneburg, Lüneburg.

FFB-DP Nr. 72

Deneke, J.F.V., 2007, Freie Berufe - Herausforderungen an die Wissenschaft, FFB-Diskussionspapier Nr. 71, Fakultät II, Wirtschafts-, Verhaltens- und Rechtswissenschaften, Universität Lüneburg, Lüneburg. FFB-DP Nr. 71

Merz, J., Böhm, P., Hanglberger, D., Stolze, H. und R. Rucha, 2007, Wann werden Serviceleistungen nachgefragt? Eine Wirkungsanalyse anhand des neuen FFB-Mikrosimulationsmodells ServSim, FFBDiskussionspapier Nr. 70, Fakultät II, Wirtschafts-, Verhaltens- und Rechtswissenschaften, Universität Lüneburg, Lüneburg.

FFB-DP Nr. 70

Stolze H., 2007, Die Nachfrage nach Gesundheitsleistungen - Ergebnisse der FFB-Patientenumfrage, FFBDiskussionspapier Nr. 69, Fakultät II, Wirtschafts-, Verhaltens- und Rechtswissenschaften, Universität Lüneburg, Lüneburg.

FFB-DP Nr. 69

Burgert, D., 2006, Betriebliche Weiterbildung und Verbleib Älterer im Betrieb, FFB-Diskussionspapier Nr. 68, Fakultät II, Wirtschafts-, Verhaltens- und Rechtswissenschaften, Universität Lüneburg, Lüneburg. FFB-DP Nr. 68

Merz, J., 2006, Polarisierung der Einkommen von Selbständigen? - Zur Dynamik der Einkommensverteilung von Freiberuflern und Unternehmern, FFB-Diskussionspapier Nr. 67, Fakultät II, Wirtschafts-, Verhaltensund Rechtswissenschaften, Universität Lüneburg, Lüneburg.

FFB-DP Nr. 67

Paic, P., 2006, Informationelle Zugänge für die empirische Untersuchung freiberuflicher Existenzgründungen, FFB-Diskussionspapier Nr. 66, Fakultät II, Wirtschafts-, Verhaltens- und Rechtswissenschaften, Universität Lüneburg, Lüneburg.

FFB-DP Nr. 66

Merz, J. und M. Zwick, 2006, Einkommensanalysen mit Steuerdaten - Mikroanalysen zu hohen Einkommen und Selbständigkeit und Mikrosimulation zu Politikalternativen der Einkommen-, Körperschaft- und Gewerbesteuer, FFB-Diskussionspapier Nr. 65, Fakultät II, Wirtschafts-, Verhaltens- und Rechtswissenschaften, Universität Lüneburg, Lüneburg.

FFB-DP Nr. 65

Merz, J., Stolze, H. und M. Zwick, 2006, Wirkungen alternativer Steuerreformmodelle auf die Einkommensverteilung von Freien und anderen Berufen, FFB-Diskussionspapier Nr. 64, Fakultät II, Wirtschafts-, Verhaltens- und Rechtswissenschaften, Universität Lüneburg, Lüneburg. ～FFB-DP Nr. 64

Zwick, M., 2006, Gemeindefinanzreform und Freie Berufe, FFB-Diskussionspapier Nr. 63, Fakultät II, Wirtschafts-, Verhaltens- und Rechtswissenschaften, Universität Lüneburg, Lüneburg. $\quad$ FFB-DP Nr. 63 
Burgert, D., 2006, Kündigungsschutz - Ein Hindernis für das Beschäftigungswachstum? FFBDiskussionspapier Nr. 62, Fakultät II, Wirtschafts-, Verhaltens- und Rechtswissenschaften, Universität Lüneburg, Lüneburg.

FFB-DP Nr. 62

Paic, P., 2006, Existenzgründung und Einkommen Freier Berufe - Ergebnisse aus dem Sozio-ökonomischen Panel und der FFB-Onlineumfrage Freie Berufe, FFB-Diskussionspapier Nr. 61, Fakultät II, Wirtschafts-, Verhaltens- und Rechtswissenschaften, Universität Lüneburg, Lüneburg.

FFB-DP Nr. 61

Merz, J. und P. Böhm, 2006, Arbeitszeit und Einkommen - Neue Ergebnisse aus der deutschen Zeitbudgeterhebung, FFB-Diskussionspapier Nr. 60, Fakultät II, Wirtschafts-, Verhaltens- und Rechtswissenschaften, Universität Lüneburg, Lüneburg.

FFB-DP Nr. 60

Merz, J., 2006, Hohe und niedrige Einkommen - Neue Ergebnisse zu Freien und anderen Berufen aus dem Armuts- und Reichtumsbericht der Bundesregierung, FFB-Diskussionspapier Nr. 59, Fakultät II, Wirtschafts-, Verhaltens- und Rechtswissenschaften, Universität Lüneburg, Lüneburg. ～FFB-DP Nr. 59

Merz, J., Vorgrimler, D. und M. Zwick, 2006, De facto Anonymised Microdata File on Income Tax Statistics 1998, FFB-Diskussionspapier Nr. 58, Fakultät II, Wirtschafts-, Verhaltens- und Rechtswissenschaften, Universität Lüneburg, Lüneburg.

FFB-DP Nr. 58

Merz, J. and L. Osberg, 2006, Keeping in Touch - A Benefit of Public Holidays. FFB-Diskussionspapier Nr. 57, Fakultät II, Wirtschafts-, Verhaltens- und Rechtswissenschaften, Universität Lüneburg, Lüneburg.

FFB-DP Nr. 57

Merz, J. and P. Paic, 2006, Start-up success of freelancers - New microeconometric evidence from the German Socio-Economic Panel. FFB-Diskussionspapier Nr. 56, Fakultät II, Wirtschafts-, Verhaltens- und Rechtswissenschaften, Universität Lüneburg, Lüneburg.

FFB-DP Nr. 56

Merz, J. und P. Paic, 2006, Erfolgsfaktoren freiberuflicher Existenzgründung - Neue mikroökonometrische Ergebnisse mit Daten des Sozio-ökonomischen Panels, FFB-Diskussionspapier Nr. 55, Fakultät II, Wirtschafts-, Verhaltens- und Rechtswissenschaften, Universität Lüneburg, Lüneburg. $\quad$ FFB-DP Nr. 55

Merz, J. and H. Stolze, 2006, Representative Time Use Data and Calibration of the American Time Use Studies 1965-1999, FFB-Diskussionspapier Nr. 54, Fakultät II, Wirtschafts-, Verhaltens- und Rechtswissenschaften, Universität Lüneburg, Lüneburg.

FFB-DP Nr. 54

Paic, P. und H. Brand, 2005, Die Freien Berufe im Sozio-ökonomischen Panel - Systematische Berichtigung der kritischen Wechsel innerhalb der Selbständigengruppe, FFB-Diskussionspapier Nr. 53, Fachbereich Wirtschafts- und Sozialwissenschaften, Universität Lüneburg, Lüneburg.

FFB-DP Nr. 53

Merz, J. und P. Paic, 2005, Zum Einkommen der Freien Berufe - Eine Ordered Probit-Analyse ihrer Determinanten auf Basis der FFB-Onlineumfrage, FFB-Diskussionspapier Nr. 52, Fachbereich Wirtschafts- und Sozialwissenschaften, Universität Lüneburg, Lüneburg.

FFB-DP Nr. 52

Burgert, D., 2005, Schwellenwerte im deutschen Kündigungsschutzrecht - Ein Beschäftigungshindernis für kleine Unternehmen?, FFB-Diskussionspapier Nr. 51, Fachbereich Wirtschafts- und Sozialwissenschaften, Universität Lüneburg, Lüneburg.

FFB-DP Nr. 51

Merz, J., Böhm, P. und D. Burgert, 2005, Arbeitszeitarrangements und Einkommensverteilung - Ein Treatment Effects Ansatz der Einkommensschätzung für Unternehmer, Freiberufler und abhängig Beschäftigte, FFBDiskussionspapier Nr. 50, Fachbereich Wirtschafts- und Sozialwissenschaften, Universität Lüneburg, Lüneburg.

FFB-DP Nr. 50

Burgert, D., 2005, The Impact of German Job Protection Legislation on Job Creation in Small Establishments An Application of the Regression Discontinuity Design, FFB-Diskussionspapier Nr. 49, Fachbereich Wirtschafts- und Sozialwissenschaften, Universität Lüneburg, Lüneburg.

FFB-DP Nr. 49

Merz, J. und D. Burgert, 2005, Arbeitszeitarrangements - Neue Ergebnisse aus der nationalen Zeitbudgeterhebung 2001/02 im Zeitvergleich, FFB-Diskussionspapier Nr. 48, Fachbereich Wirtschaftsund Sozialwissenschaften, Universität Lüneburg, Lüneburg.

FFB-DP Nr. 48

Merz, J., Böhm, P. and D. Burgert, 2005, Timing, Fragmentation of Work and Income Inequality - An Earnings Treatment Effects Approach, FFB-Diskussionspapier Nr. 47, Fachbereich Wirtschafts- und Sozialwissenschaften, Universität Lüneburg, Lüneburg.

FFB-DP Nr. 47 
Merz, J. und P. Paic, 2004, Existenzgründungen von Freiberuflern und Unternehmer - Eine Mikroanalyse mit dem Sozio-ökonomischen Panel FFB-Diskussionspapier Nr. 46, Fachbereich Wirtschafts- und Sozialwissenschaften, Universität Lüneburg, Lüneburg.

FFB-DP Nr. 46

Merz, J. und D. Burgert, 2004, Wer arbeitet wann? Arbeitszeitarrangements von Selbständigen und abhängig Beschäftigten - Eine mikroökonometrische Analyse deutscher Zeitbudgetdaten, FFB-Diskussionspapier Nr. 45, Fachbereich Wirtschafts- und Sozialwissenschaften, Universität Lüneburg, Lüneburg.

FFB-DP Nr. 45

Hirschel, D. und J. Merz, 2004, Was erklärt hohe Arbeitseinkommen der Selbständigen - Eine Mikroanalyse mit Daten des Sozio-ökonomsichen Panels, FFB-Diskussionspapier Nr. 44, Fachbereich Wirtschafts- und Sozialwissenschaften, Universität Lüneburg, Lüneburg.

FFB-DP Nr. 44

Ackermann, D., Merz, J. und H. Stolze, 2004, Erfolg und Erfolgsfaktoren freiberuflich tätiger Ärzte Ergebnisse der FFB-Ärztebefragung für Niedersachsen, FFB-Diskussionspapier Nr. 43, Fachbereich Wirtschafts- und Sozialwissenschaften, Universität Lüneburg, Lüneburg.

FFB-DP Nr. 43

Hirschel, D., 2003, Do high incomes reflect individual performance? The determinants of high incomes in Germany, FFB-Diskussionspapier Nr. 42, Department of Economics and Social Sciences, University of Lüneburg, Lüneburg.

FFB-DP Nr. 42

Merz, J. and D. Burgert, 2003, Working Hour Arrangements and Working Hours - A Microeconometric Analysis Based on German Time Diary Data, FFB-Diskussionspapier Nr. 41, Department of Economics and Social Sciences, University of Lüneburg, Lüneburg.

FFB-DP Nr. 41

Merz, J. und M. Zwick, 2002, Hohe Einkommen: Eine Verteilungsanalyse für Freie Berufe, Unternehmer und abhängig Beschäftigte, Eine Mikroanalyse auf der Basis der Einkommensteuerstatistik, FFBDiskussionspapier Nr. 40, Fachbereich Wirtschafts- und Sozialwissenschaften, Universität Lüneburg, Lüneburg.

FFB-DP Nr. 40

Merz, J. and D. Hirschel, 2003, The distribution and re-distribution of income of self-employed as freelancers and entrepreneurs in Europe, FFB-Diskussionspapier Nr. 39, Department of Economics and Social Sciences, University of Lüneburg, Lüneburg.

FFB-DP Nr. 39

Stolze, H., 2002, Datenbankbankgestützte Internetpräsenzen - Entwicklung und Realisation am Beispiel der Homepage des Forschungsinstituts Freie Berufe (FFB) der Universität Lüneburg http://ffb.unilueneburg.de, FFB-Diskussionspapier Nr. 38, Fachbereich Wirtschafts- und Sozialwissenschaften, Universität Lüneburg, Lüneburg.

FFB-DP Nr. 38

Merz, J., 2002, Zur Kumulation von Haushaltsstichproben, FFB-Diskussionspapier Nr. 37, Fachbereich Wirtschafts- und Sozialwissenschaften, Universität Lüneburg, Lüneburg.

FFB-DP Nr. 37

Merz, J., 2002, Reichtum in Deutschland: Hohe Einkommen, ihre Struktur und Verteilung - Eine Mikroanalyse mit der Einkommensteuerstatistik für Selbständige und abhängig Beschäftigte, FFB-Diskussionspapier Nr. 36, Fachbereich Wirtschafts- und Sozialwissenschaften, Universität Lüneburg, Lüneburg. FFB-DP Nr. 36

Merz, J. und M. Zwick, 2002, Verteilungswirkungen der Steuerreform 2000/2005 im Vergleich zum ,Karlsruher Entwurf Auswirkungen auf die Einkommensverteilung bei Selbständigen (Freie Berufe, Unternehmer und abhängig Beschäftigte), FFB-Diskussionspapier Nr. 35, Fachbereich Wirtschafts- und Sozialwissenschaften, Universität Lüneburg, Lüneburg.

FFB-DP Nr. 35

Merz, J., Stolze, H. and M. Zwick, 2002, Professions, entrepreneurs, employees and the new German tax (cut) reform 2000 - A MICSIM microsimulation analysis of distributional impacts, FFB-Diskussionspapier Nr. 34, Department of Economics and Social Sciences, University of Lüneburg, Lüneburg. $\quad$ FFB-DP Nr. 34

Forschungsinstitut Freie Berufe, 2002, Freie Berufe im Wandel der Märkte - 10 Jahre Forschungsinstitut Freie Berufe (FFB) der Universität Lüneburg, Empfang am 4. November 1999 im Rathaus zu Lüneburg, FFBDiskussionspapier Nr. 33, Fachbereich Wirtschafts- und Sozialwissenschaften, Universität Lüneburg, Lüneburg.

FFB-DP Nr. 33

Merz, J., 2002, Time Use Research and Time Use Data - Actual Topics and New Frontiers, FFB-Discussion Paper No. 32, Department of Economics and Social Sciences, University of Lüneburg, Lüneburg.

FFB-DP Nr. 32 
Merz, J., 2001, Freie Berufe im Wandel der Arbeitsmärkte, FFB-Diskussionspapier Nr. 31, Fachbereich Wirtschafts-und Sozialwissenschaften, Universität Lüneburg, Lüneburg.

FFB-DP Nr. 31

Merz, J., 2001, Was fehlt in der EVS? Eine Verteilungsanalyse hoher Einkommen mit der verknüpften Einkommensteuerstatistik für Selbständige und abhängig Beschäftigte, FFB-Diskussionspapier Nr. 30, Fachbereich Wirtschafts- und Sozialwissenschaften, Universität Lüneburg, Lüneburg.

FFB-DP Nr. 30

Merz, J., 2001, Informationsfeld Zeitverwendung - Expertise für die Kommission zur Verbesserung der informationellen Infrastruktur zwischen Wissenschaft und Statistik, FFB-Diskussionspapier Nr. 29, Fachbereich Wirtschafts- und Sozialwissenschaften, Universität Lüneburg, Lüneburg.

FFB-DP Nr. 29

Schatz, C. und J. Merz, 2000, Die Rentenreform in der Diskussion - Ein Mikrosimulationsmodell für die Altersvorsorge in Deutschland (AVID-PTO), FFB-Diskussionspapier Nr. 28, Fachbereich Wirtschafts- und Sozialwissenschaften, Universität Lüneburg, Lüneburg.

FFB-DP Nr. 28

Merz, J., 2000, The Distribution of Income of Self-employed, Entrepreneurs and Professions as Revealed from Micro Income Tax Statistics in Germany, FFB-Discussion Paper No. 27, Department of Economics and Social Sciences, University of Lüneburg, Lüneburg.

FFB-DP Nr. 27

Merz, J., Loest, O. und A. Simon, 1999, Existenzgründung - Wie werde ich selbständig, wie werde ich Freiberufler? Ein Leitfaden, FFB-Diskussionspapier Nr. 26, Fachbereich Wirtschafts- und Sozialwissenschaften, Universität Lüneburg, Lüneburg.

FFB-DP Nr. 26

Merz, J. und D. Kirsten, 1998, Extended Income Inequality and Poverty Dynamics of Labour Market and Valued Household Acitivities - A Ten Years Panelanalysis for Professions, Entrepreneurs and Employees in Germany, FFB-Discussion Paper No. 25, Department of Economics and Social Sciences, University of Lüneburg, Lüneburg.

FFB-DP Nr. 25

Merz, J., Quiel, T. und K. Venkatarama, 1998, Wer bezahlt die Steuern? - Eine Untersuchung der Steuerbelastung und der Einkommenssituation für Freie und andere Berufe, FFB-Diskussionspapier Nr. 24, Fachbereich Wirtschafts- und Sozialwissenschaften, Universität Lüneburg, Lüneburg. ～FFB-DP Nr. 24

Merz, J. and R. Lang, 1997, Preferred vs. Actual Working Hours - A Ten Paneleconometric Analysis for Professions, Entrepreneurs and Employees in Germany, FFB-Discussion Paper No. 23, Department of Economics and Social Sciences, University of Lüneburg, Lüneburg.

FFB-DP Nr. 23

Merz, J., 1997, Privatisierung und Deregulierung und Freie und staatlich gebundene Freie Berufe - Einige ökonomische Aspekte, FFB-Diskussionspapier Nr. 22, Fachbereich Wirtschafts- und Sozialwissenschaften, Universität Lüneburg, Lüneburg.

FFB-DP Nr. 22

de Kam, C. A., de Haan, J., Giles, C., Manresa, A., Berenguer, E., Calonge, S., Merz, J. and K. Venkatarama, 1996, The Distribution of Effetcive Tax Burdens in Four EU Countries, FFB-Discussion Paper No. 21, Department of Economics and Social Sciences, University of Lüneburg, Lüneburg.

FFB-DP Nr. 21

Deneke, J. F. V., 1996, Freie Berufe und Mittelstand - Festrede zur Verleihung der Ehrendoktorwürde, FFBDiskussionspapier Nr. 20, Fachbereich Wirtschafts- und Sozialwissenschaften, Universität Lüneburg, Lüneburg.

FFB-DP Nr. 20

Merz, J., 1996, Die Freien Berufe - Laudatio zur Verleihung der Ehrendokotorwürde des Fachbereiches Wirtschafts- und Sozialwissenschaften der Universität Lüneburg an Prof. J. F. Volrad Deneke, FFBDiskussionspapier Nr. 19, Fachbereich Wirtschafts- und Sozialwissenschaften, Universität Lüneburg, Lüneburg.

FFB-DP Nr. 19

de Kam, C. A., de Haan, J., Giles, C., Manresa, A., Berenguer, E., Calonge, S. and J. Merz, 1996, Who pays the taxes?, FFB-Discussion Paper No. 18, Department of Economics and Social Sciences, University of Lüneburg, Lüneburg.

FFB-DP Nr. 18

Merz, J., 1996, Schattenwirtschaft und ihre Bedeutung für den Arbeitsmarkt, FFB-Diskussionspapier Nr. 17, Fachbereich Wirtschafts- und Sozialwissenschaften, Universität Lüneburg, Lüneburg.

FFB-DP Nr. 17

Merz, J. und D. Kirsten, 1995, Freie Berufe im Mikrozensus II - Einkommen und Einkommensverteilung anhand der ersten Ergebnisse für die neuen und alten Bundesländer 1991, FFB-Diskussionspapier Nr. 16, Fachbereich Wirtschafts- und Sozialwissenschaften, Universität Lüneburg, Lüneburg. FFB-DP Nr. 16 
Merz, J. und D. Kirsten, 1995, Freie Berufe im Mikrozensus I - Struktur und quantitative Bedeutung anhand der ersten Ergebnisse für die neuen und alten Bundesländer 1991, FFB-Diskussionspapier Nr. 15, Fachbereich Wirtschafts- und Sozialwissenschaften, Universität Lüneburg, Lüneburg.

FFB-DP Nr. 15

Merz, J., 1995, MICSIM - Concept, Developments and Applications of a PC-Microsimulation Model for Research and Teaching, FFB-Discussion Paper No. 14, Department of Economics and Social Sciences, University of Lüneburg, Lüneburg.

FFB-DP Nr. 14

Rönnau, A., 1995, Freie Berufe in der DDR, der Bundesrepublik Deutschland und im wiedervereinten Deutschland: Auswertungen von Berufstätigenerhebung und Arbeitsstättenzählung, FFBDiskussionspapier Nr. 13, Fachbereich Wirtschafts- und Sozialwissenschaften, Universität Lüneburg, Lüneburg.

FFB-DP Nr. 13

Burkhauser, R. V., Smeeding, T. M. and J. Merz, 1994, Relative Inequality and Poverty in Germany and the United States Using Alternative Equivalence Scales, FFB-Discussion Paper No. 12, Department of Economics and Social Sciences, University of Lüneburg, Lüneburg.

FFB-DP Nr. 12

Widmaier, U., Niggemann, H. and J. Merz, 1994, What makes the Difference between Unsuccessful and Successful Firms in the German Mechanical Engineering Industry? A Microsimulation Approach Using Data from the NIFA-Panel, FFB-Discussion Paper No. 11, Department of Economics and Social Sciences, University of Lüneburg, Lüneburg.

FFB-DP Nr. 11

Merz, J., 1994, Microdata Adjustment by the Minimum Information Loss Principle, FFB-Discussion Paper No. 10, Department of Economics and Social Sciences, University of Lüneburg, Lüneburg. $\quad$ FFB-DP Nr. 10

Merz, J., 1994, Microsimulation - A Survey of Methods and Applications for Analyzing Economic and Social Policy, FFB-Discussion Paper No. 9, Department of Economics and Social Sciences, University of Lüneburg, Lüneburg. FFB-DP Nr. 9

Merz, J., Garner, T., Smeeding, T. M., Faik, J. and D. Johnson, 1994, Two Scales, One Methodology Expenditure Based Equivalence Scales for the United States and Germany, FFB-Discussion Paper No. 8, Department of Economics and Social Sciences, University of Lüneburg, Lüneburg.

FFB-DP Nr. 8

Krickhahn, T., 1993, Lobbyismus und Mittelstand: Zur Identifikation der Interessenverbände des Mittelstands in der Bundesrepublik Deutschland, FFB-Diskussionspapier Nr. 7, Fachbereich Wirtschafts- und Sozialwissenschaften, Universität Lüneburg, Lüneburg.

FFB-DP Nr. 7

Merz, J., 1993, Market and Non-Market Labor Supply and Recent German Tax Reform Impacts - Behavioral Response in a Combined Dynamic and Static Microsimulation Model, FFB-Discussion Paper No. 6, Department of Economics and Social Sciences, University of Lüneburg, Lüneburg. $\quad$ FFB-DP Nr. 6

Merz, J., 1993, Microsimulation as an Instrument to Evaluate Economic and Social Programmes, FFBDiscussion Paper No. 5, Department of Economics and Social Sciences, University of Lüneburg, Lüneburg.

FFB-DP Nr. 5

Merz, J., 1993, Statistik und Freie Berufe im Rahmen einer empirischen Wirtschafts- und Sozialforschung, Antrittsvorlesung im Fachbereich Wirtschafts- und Sozialwissenschaften der Universität Lüneburg, FFBDiskussionspapier Nr. 4, Fachbereich Wirtschafts- und Sozialwissenschaften, Universität Lüneburg, Lüneburg.

FFB-DP Nr. 4

Merz, J. and J. Faik, 1992, Equivalence Scales Based on Revealed Preference Consumption Expenditure Microdata - The Case of West Germany, FFB-Discussion Paper No. 3, Department of Economics and Social Sciences, University of Lüneburg, Lüneburg.

FFB-DP Nr. 3

Merz, J., 1992, Time Use Dynamics in Paid Work and Household Activities of Married Women - A Panel Analysis with Household Information and Regional Labour Demand, FFB-Discussion Paper No. 2, Department of Economics and Social Sciences, University of Lüneburg, Lüneburg.

FFB-DP Nr. 2

Forschungsinstitut Freie Berufe, 1992, Festliche Einweihung des Forschungsinstituts Freie Berufe am 16. Dezember 1991 im Rathaus zu Lüneburg, FFB-Diskussionspapier Nr. 1, Fachbereich Wirtschafts- und Sozialwissenschaften, Universität Lüneburg, Lüneburg.

FFB-DP Nr. 1 
FFB-Jahresberichte und FFB-Reprints werden auf Anfrage kostenlos zugesandt.

FFB-Diskussionspapiere und FFB-Dokumentationen können über unsere FFB-Homepage kostenlos heruntergeladen werden.

FFB-Bücher können sowohl bei uns als auch beim NOMOS Verlag bestellt werden. Sonstige Arbeitsberichte und Sonstige Bücher sind über den jeweiligen Autor zu beziehen.

www.leuphana.de/ffb 